\title{
GUP1 and its close homologue GUP2, encoding multi-membrane- spanning proteins involved in active glycerol uptake in Saccharomyces cerevisiae
}

\author{
Bjørn Holst ${ }^{1,3}$, Christina Lunde ${ }^{1,4}$, Fernanda Lages $^{2}$, Rui Oliveira ${ }^{2}$, Cândida Lucas ${ }^{2}$ and \\ Morten C. Kielland-Brandt ${ }^{1}$, * \\ ${ }^{1}$ Department of Yeast Genetics, Carlsberg Laboratory, Gamle Carlsberg Vej 10, DK-2500 \\ Copenhagen Valby, Denmark, ${ }^{2}$ Department of Biology - CCA, University of Minho, 4709 Braga \\ Codex, Portugal.
}

Running title: Proteins involved in active glycerol transport in yeast

Key words: Glycerol, permeases, Saccharomyces cerevisiae, GUP1, GUP2

\author{
Author for correspondence and proofs: \\ Morten C. Kielland-Brandt \\ Department of Yeast Genetics \\ Carlsberg Laboratory \\ Gamle Carlsberg Vej 10 \\ DK-2500 Copenhagen Valby \\ Denmark \\ Phone (+45) 33275331 \\ Fax (+45) 33274765 \\ mkb@crc.dk
}

\footnotetext{
${ }^{3}$ Present address: Institute for Food Safety and Toxicology, Danish Veterinary and Food Administration, Mørkhøj Bygade 19, DK-2860 Søborg, Denmark,

4 Present address: Plant Biochemistry Laboratory, Department of Plant Biology, The Royal Veterinary and Agricultural University, Thorvaldsensvej 40, DK-1871 Frederiksberg C, Copenhagen, Denmark.

* Corresponding author
} 


\section{Summary}

Many yeast species can utilise glycerol, both as sole carbon source and as an osmolyte. In Sacharomyces cerevisiae, physiological studies have previously shown the presence of an active uptake system driven by electrogenic proton symport. We have used transposon mutagenesis to isolate mutants affected in the transport of glycerol into the cell. Here we present the identification of $Y G L 084 c$, encoding a multi-membrane-spanning protein, as being essential for proton symport of glycerol into Saccharomyces cerevisiae. The gene is named GUP1 (Glycerol UPtake) and is important for growth on glycerol as carbon and energy source, as well as for osmotic protection by added glycerol, of a strain deficient in glycerol production. Another ORF, YPL189w, presenting a high degree of homology to YGLO84c, similarly appears to be involved in active glycerol uptake in salt-containing glucose-based media in strains deficient in glycerol production. Analogously, this gene is named GUP2. To our knowledge, this is the first report on a gene product involved in active transport of glycerol in yeasts. Mutations with the same phenotypes occurred in two other open reading frames of previously unknown function, YDLO74c and YPL180w.

\section{Introduction}

Osmotic stress is known to trigger a set of cellular responses which enable Saccharomyces cerevisiae to adapt to changes in the environment (Varela and Mager, 1996). When S. cerevisiae is exposed to osmotic stress, it responds by accumulating glycerol in the cytoplasm, up to molar concentrations, which counteracts cell dehydration. Glycerol is synthesised from dihydroxyacetone phosphate in two steps that branch off from glycolysis or gluconeogenesis. In the first step, dihydroxyacetone phosphate is converted into glycerol 3-phosphate in a reaction catalysed by a NAD-dependent glycerol 3-phosphate dehydrogenase. Two isoenzymes exist, encoded by GDP1 and GPD2 (Larsson et al., 1993; Albertyn et al., 1994; Eriksson et al., 1995); however, only expression of GPD1 is stimulated by osmotic stress. Similarly, two isoenzyme forms exist for the glycerol 3-phosphate-specific phosphatase, encoded by GPP1 and GPP2 (Norbeck et al., 1996), which catalyses the final formation of glycerol. Also in this case only one of the genes, namely $G P P 2$, is under osmotic regulation. Strains deleted for both GPD1 and GPD2 can no longer 
synthesise glycerol, and they become highly sensitive to osmotic stress. However, Albertyn et al. (1994) observed that a gpdl strain upon salt stress is able to increase the intracellular glycerol level by taking up extracellular glycerol against a concentration gradient, indicating that the cell has an active uptake system for glycerol. Furthermore, R. Ansell and S. Hohmann (personal communication) found that the presence of small amounts of glycerol in the medium lowers the osmo-sensitivity of strains deficient in glycerol synthesis. This observation makes an important basis for screening for mutants deficient in active glycerol uptake, used in the present work.

$S$. cerevisiae is also able to utilise glycerol as sole source of carbon. The catabolic pathway involves a glycerol kinase encoded by GUT1 (Sprague and Cronan, 1977; Rønnow, 1992; Pavlik et al., 1993), and a mitochondrial glycerol 3-phosphate dehydrogenase encoded by GUT2 (Sprague and Cronan, 1977; Rønnow and Kielland-Brandt, 1993). Mutants lacking one of these genes are not able to use glycerol as carbon source. Some yeast species, including Schizosaccharomyces pombe, have an alternative catabolic pathway (May et al., 1982; Gancedo et al., 1986), in which glycerol is first converted into dihydroxyacetone by a dehydrogenase and further to dihydroxyacetone phosphate by a kinase. Until recently, this pathway was thought not to be present in S. cerevisiae. However, results by Norbeck and Blomberg (1997) and Blomberg (1997) suggest that these enzymes might also exist in $S$. cerevisiae, although their function is unknown.

Physiological characterisation of glycerol uptake in S. cerevisiae has shown that glycerol enters the cell by at least two different mechanisms depending on the growth conditions. When glucose is present, glycerol can enter the cells by facilitated transport, probably through the glycerol channel, encoded by the FPS1 gene (Van Aelst et al., 1991; Luyten et al., 1995; Sutherland et al., 1997; Tamas et al, 1999). However, in ethanol-, glycerol- and acetate-grown cells, a proton symport system ensures uptake of glycerol (Lages and Lucas, 1997; Sutherland et al., 1997).

To understand the mechanisms behind glycerol uptake in S. cerevisiae, as well as to identify other proteins important for glycerol catabolism, we isolated mutants deficient in uptake and/or catabolism of glycerol, using a parental strain unable to produce glycerol. With a mTn-lacZ/LEU2mutagenised library set-up (Ross-Macdonald et al., 1995), mutants were isolated by two different screens. In the first screen, mutants that were no longer able to utilise glycerol as sole carbon source were selected. In the second screen we isolated mutants which lack the ability to survive salt stress even when glycerol is available in the medium. This screen was included in order to select mutants directly affected in genes encoding proteins important for the active uptake of glycerol. We isolated and identified mutants in four different loci, of which only one, SPT7, has previously been assigned 
a function in the utilisation of glycerol as carbon source (Winston et al., 1984). The three other ORF's all encode unknown putative proteins. One of these, $Y G L 084 \mathrm{c}$, has been suggested to encode a multi-membrane-spanning protein belonging to the major facilitator superfamily (Nelissen et al., 1997). Our results show that YGL084c is essential for proper growth on glycerol, as well as for glycerol-mediated recovery from salt stress. A highly homologous ORF, YPL189w, turned out to be important for glycerol uptake in glucose-grown cells when exposed to salt stress. We propose that both genes encode proteins which have the ability to actively transport glycerol into the cell, $Y G L 084 c$, in particular, being responsible for the previously described glycerol-proton symporter activity in S. cerevisiae.

\section{Results}

Isolation of mutants defective in uptake/catabolism of glycerol

To identify proteins involved in active uptake of glycerol, as well as to identify novel proteins necessary for the catabolism of glycerol, we subjected a gpd1 gpd2 mutant (YSH6.142-3D), which lacks both isoenzymes of cytoplasmic glycerol 3-phosphate dehydrogenases, and is therefore unable to produce glycerol, to transposon mutagenesis. The parental strain was transformed with a mixture of yeast genomic fragments cut out from an mTn-lacZ/LEU2-mutagenised plasmid library (RossMacdonald et al., 1995; see Experimental procedures). The efficiency of yeast to integrate linear DNA into homologous regions of the genome ensures that each integrant becomes a mutant having a transposon somewhere in the genome, quite often disrupting a coding region. Two different screens were used to select mutants of interest. In one, we picked mutants that were no longer able to grow with $2 \%$ glycerol as sole carbon and energy source ( $g u t$-diagnostic medium). In the second screen, we isolated mutants lacking glycerol-mediated recovery from salt stress on rich medium with $1 \mathrm{M} \mathrm{NaCl}$, containing glucose as carbon source and supplemented with $10 \mathrm{mM}$ glycerol. After transformation in the gpd1 gpd2 background with the inserts of the Tn-library, around 10,000 colonies were isolated on synthetic complete medium without leucine, selecting for integration of the transposon. All colonies were subsequently screened for both phenotypes by replica plating to both gut-diagnostic plates, containing glycerol as sole carbon source, and to YPD plates containing $1 \mathrm{M} \mathrm{NaCl}$ and $10 \mathrm{mM}$ glycerol. After several repetitive rounds of screening in both set-ups and 
elimination of mutants with a general growth phenotype, we ended up with 5 mutants (Table 1), satisfying the criteria of both screens, i.e. all five mutants both lack glycerol-mediated recovery from salt stress and have reduced ability to utilise glycerol as carbon source (gut) (Fig. 1). None of the mutants were petites as they all grow with lactate and ethanol as carbon source (data not shown). Using a so-called rescue plasmid set-up (Ross-Macdonald et al., 1995), we recovered transposon sequences together with sequences from the flanking regions, thereby identifying four genes that were disrupted by the transposon: SPT7, YDL074c, YPL180w and YGL084c (GUPI) (Table 1). Only SPT7 has earlier been assigned a function (Winston et al., 1987).

In transposon mutant BHY23, the transposon had been inserted approximately in the middle of SPT7. The gene encodes a 1332 amino acid residue nuclear protein. SPT7 is one of the SPT genes, originally identified by mutations acting as suppressors of certain $T y$ insertions which affect transcription start site selection (Winston et al., 1984; Gansheroff et al., 1995). spt7 mutants are known to grow slowly on several carbon sources, including glycerol.

The three other Tn-tagged ORF's had not been assigned any function yet. YDLO74c encodes a 700 amino acid residue putative protein. It was recovered twice, through BHY13 and BHY24, with the transposon inserted at the same position, namely after nucleotide 1463. Curiously, the two mutants show slightly different phenotype, suggesting a second mutation in one of them. Search in the databases suggests some homology between Yd1074cp and proteins engaged in interaction with microtubules. In BHY21, the transposon is located in the very C-terminal end of ORF YPL180w, which encodes a 799 amino acid residue putative protein. YPL180w shows no significant homology to any protein in the databases.

In mutant BHY22, the transposon is located in the N-terminal end of an open reading frame, $Y G L 084 c$, encoding a 560 amino acid residue putative protein. YGL084c has recently been suggested to encode a multi-membrane-spanning protein of unknown function, belonging to the major facilitator superfamily (Nelissen et al., 1997). The predicted transmembrane nature of Yg1084cp and the observed phenotypes of BHY22 make Yg1084cp an obvious candidate for an active glycerol transporter, or perhaps a sensor. The reason for this lies in the fact that the mutant affected in this gene is deficient in both glycerol-mediated recovery from salt stress and in growth on glycerol, indicating a role in uptake. Therefore we called the gene GUP1, for Glycerol UPtake. The name has been reserved in the Saccharomyces Genome Database in Stanford. Blast searches in the databases revealed two proteins with significant homology to the predicted amino acid sequence of Ygl084cp, namely Ypl189wp, a 608 amino acid residue putative protein also from S. cerevisiae, 
and the one encoded by SPAC24H6.01c, an ORF of 231 codons from Schiz. pombe. At the amino acid level the sequence identity between $Y G L 084 \mathrm{c}$ and $Y P L 189 \mathrm{w}$ is $57 \%$ and the similarity is $77 \%$, suggesting closely related functions (Fig. 2, panel A). The somewhat smaller putative ORF from Schiz. pombe is homologous (49\% identity) to the N-terminal part of YGL084c (Fig. 2, panel A). All three proteins are predicted to be membrane proteins, Yg1084cp and Ypl189wp having 8-10 putative membrane-spanning domains, and Spac24H6.01 cp only four. By comparing the hydrophobicity plots (Kyte and Doolittle, 1982) of these proteins, the similarity is also quite obvious (Fig. 2, panel B).

\section{Phenotypes of gup1}

To analyse further the phenotype of gupl, we cloned a chromosomal copy of GUP1 with 1421 nucleotides of the promoter region, using homologous recombination/GAP-repair (Orr-Weaver and Szostak, 1983; Rothstein, 1991; see Experimental procedures). By using this strategy we ensured cloning with the high fidelity characteristic of in vivo replication. As can be seen in Fig. 3 (panel A), introduction of a low-copy (centromere-based) plasmid ( $\mathrm{pBH} 2178$ ) containing GUP1 into strain BHY29-2C complements both the gut (glycerol utilisation) phenotype and the ability to recover from salt stress when glycerol is present in the medium. Over-expression of GUPI from a highcopy $(2 \mu$-based) plasmid does not lead to enhanced growth on gut-diagnostic plates (glycerol as carbon and energy source), or on YPD containing $1 \mathrm{M} \mathrm{NaCl}$ and supplemented with $10 \mathrm{mM}$ glycerol. However, the zone in which the cells can grow on YPD $+1 \mathrm{M} \mathrm{NaCl}$, around a filter disk containing glycerol, is clearly enlarged when a high-copy plasmid with GUP1 has been introduced into BHY29-2C cells, as compared to the low-copy situation (Fig. 3, panel B). To see if this ability also results in a general higher osmo-tolerance, growth experiments on plates with rich medium with increasing salt concentration were performed. However, there was no improved osmotolerance when GUP1 was overexpressed, relative to expression from a low-copy plasmid (data not shown).

To analyse the effect of a gup1 deletion in an otherwise wild-type background, we next made a one-step deletion/disruption of GUPl by insertion of the his $5^{+}$gene from Schiz. pombe, as described in Experimental procedures. The phenotypes of the resulting strain, BHY54, on different media were analysed by a drop test, where dilution series of cells are spotted on plates (Fig. 4). 
Whereas there was no effect when lactate/ethanol were tested (data not shown), deletion of GUP1 gave a clear phenotype on glycerol (Fig. 4, upper panel), implying the importance of Guplp in the utilisation of glycerol as carbon source, and not a common defect in utilising non-fermentable carbon sources. Unexpectedly, we also saw a phenotype when the gupl mutant was growing with glucose as carbon source in the presence of osmotic stress (Fig. 4, lower panel). This phenotype was more pronounced when the cells were exposed to osmotic stress with $1 \mathrm{M} \mathrm{NaCl}$ than with $1 \mathrm{M} \mathrm{KCl}$ (data not shown). Addition of $50 \mathrm{mM}$ glycerol to the $1 \mathrm{M} \mathrm{NaCl}$ plates did not remedy the phenotype. Sorbitol, 1.5 M, leading to about the same decrease in water activity, had almost no effect on growth (data not shown). Introduction of a low-copy plasmid with GUP1 under control of its own promoter into a gup1 strain restores wild-type growth in all cases (Fig. 4 and data not shown). These observations suggest that even in a wild-type background, i.e. with no impairment in glycerol synthesis, Guplp, and thus probably also some (re)uptake of glycerol, is necessary for proper growth under osmotic stress. However, it should be kept in mind that the effect of lacking Gup1p is much more dramatic when glycerol synthesis is blocked (Fig. 3A, lower panel, lane 2). Introduction of a multi-copy plasmid with GUP1 into BHY54 did not give any enhanced growth, as compared to a single-copy plasmid with GUP1, on any of the media tested (Fig. 4 and data not shown).

\section{Deletion of YPL189w}

As previously mentioned, screening of the Saccharomyces Genome Database revealed Ypl189wp to be a close homologue of Gup1p. The high degree of identity, 57\% at the amino acid level, suggests that the homologue might serve a very similar function in the cell. To study the function of Ypl189wp, we made deletion mutant strains in which the coding region, except for the first 10 amino acid residues, was replaced by the heterologous marker for geneticin resistance, $\operatorname{kanMX}$ (Wach et al., 1994). The deletion was made in both W303-1A and BHY54, which already lacks GUP1. The phenotypes of the deletion mutants (CLy5 and CLy3, respectively) were tested by drop assays on several different carbon sources. Deletion of YPL189w did not cause any impairment of growth when tested in glucose, glycerol or ethanol, as compared with the corresponding parental strains (data not shown). Also when exposed to osmotic stress on glucose-based media, by the addition of $1 \mathrm{M} \mathrm{NaCl}, 1 \mathrm{M} \mathrm{KCl}$ or $1.5 \mathrm{M}$ sorbitol, no effects were seen. In liquid media with glucose, 
ethanol or glycerol as sole carbon source, deletion of YPL189w did not cause significant changes in the specific growth rate (data not shown).

We also tested the ability of YPL189w to complement a deletion of GUP1. Using PCR with chromosomal DNA as template, followed by homologous recombination/GAP-repair, we cloned YPL189w with its 789-bp promoter region and inserted it into both a centromere-based vector and a $2 \mu$-based vector. However, neither on gut-diagnostic plates nor on YPD plates containing $1 \mathrm{M} \mathrm{NaCl}$ was overexpression of YPL189w able to complement the deletion phenotypes of the gup 1 mutant (data not shown).

It should be noted that these experiments were carried out with a strain with functional glycerol synthesis. Later experiments, to be described below, revealed a phenotype for ypl189w in a strain defective for gpdl, gpd2 and gupl.

Involvement of Guplp in active transport of glycerol in ethanol-grown cells

Earlier studies on the transport of glycerol in ethanol-grown cells showed that active glycerol uptake in S. cerevisiae is accompanied by a simultaneous uptake of protons (Lages and Lucas, 1997; Sutherland et al., 1997). Both studies showed that active glycerol uptake was only seen in glucose-derepressed cells. In glucose-grown cells, on the other hand, glycerol enters the cell through the constitutively expressed Fps1p, which has been suggested to mediate glycerol uptake by facilitated diffusion (Sutherland et al., 1997), and which responds to salt stress by closing, thereby causing glycerol retention (Tamás et al., 1999). Under both repressing and derepressing conditions, simple diffusion through the membrane with a similar passive diffusion coefficient was also measured. The phenotypes of the gup 1 mutant isolated here suggested that Gup1p could be responsible for the active uptake of glycerol. Therefore, we analysed the uptake of glycerol in both the parental strain W303-1A and the gup 1 mutant after growth on ethanol-based complex medium. Remarkably, Eadie-Hofstee plots of the glycerol uptake in both the wild-type and the gupl mutant showed that a saturable uptake system was still apparent after deletion of GUP1, with an approximately similar $\mathrm{K}_{\mathrm{m}}$ and with the $\mathrm{V}_{\max }$ only decreased around 35\% (Fig. 5; Table 2). No further significant changes were seen when the gupl mutation was combined with either a deletion of the gene for the glycerol channel, FPS1, or a deletion of the gene for the close homologue of Gup1p, YPL189w. However, the glycerol-induced proton signal was not detectable in any of the 
three gupl mutant strains (data not shown). Furthermore, the addition of the protonophore CCCP did not prevent the residual saturable glycerol uptake. Although the $V_{\max }$ for glycerol uptake in the gupl mutant strain grown on ethanol is still around $65 \%$ of the wild-type level, the defect in uptake is obviously sufficient to affect the utilisation of glycerol as carbon and energy source. One explanation for the residual glycerol uptake in the gupl mutant could be that there exists an additional uptake system with Michaelis-Menten kinetics. Another explanation could be that the residual uptake is not active as such, but is pulled by an active catabolism of glycerol under the derepressing conditions. Glycerol destined for utilisation is initially phosphorylated by a glycerol kinase, encoded by GUT1 (Sprague and Cronan, 1977; Pavlik et al., 1993). Consistently, the levels of kinase activity measured in extracts from either W303-1A or gpd1 $\Delta$ gpd2 $\Delta$ strains, as well as in extracts from gup $1 \Delta$ and gup $1 \Delta y$ pl189w $\Delta$ strains were high, $66.1 \pm 4.1$ and $55.8 \pm 11.4 \mathrm{mU} \mathrm{mg}$ $\operatorname{protein}^{-1}$ for the first two strains and a somewhat lower activity of $43.1 \pm 5.8$ and $48.9 \pm 13.5 \mathrm{mU} \mathrm{mg}$ protein $^{-1}$ for the last two strains, respectively. In order to clarify whether glycerol kinase has an effect on the apparent kinetics of uptake, we deleted GUTI both in a wild type and a gpdl mutant background (Fig 5; Table 2). In both these strains lacking GUT1, $\mathrm{V}_{\max }$ for glycerol uptake is decreased relative to the wild type level ( $26 \%$ and $11 \%$, respectively), indicating that the kinase to some extent affects the uptake kinetics. We also deleted GUP1 in different combinations with strains already lacking GUT1 and compared kinetics for the glycerol uptake under derepressing conditions (Fig. 5; Table 2). Interestingly, in neither the gut1 gpdl gupl nor the gut1 ypl189w gup 1 strains, any saturable uptake can be measured, leaving only simple diffusion. This indicates that the glycerol uptake measured in W303-1A, besides being a mixture of active uptake and passive diffusion, also is kinetically influenced by the first step in catabolism.

To investigate if other proteins which are known to be involved in glycerol metabolism are important for uptake of glycerol under derepressing conditions, we characterised uptake in strains lacking these enzymes (Table 3). The only strains with a measurable decrease in $\mathrm{V}_{\text {max }}$ appear to be the gut 1 strain and the gut 2 strain. Thus, the saturability of glycerol uptake seen in gup 1 mutants, as seen in Table 2 and Fig. 5, appears to be a consequence of the catabolism of glycerol only.

One may wonder how the relatively modest decrease in uptake in a gup 1 mutant allowed us to pick it up in the glycerol utilisation screen. We have not done much to answer this question, but it may be noted that the media are not identical; the plates are mainly defined, containing only $0.05 \%$ peptone, while the growth media for uptake assays are complex.

We also analysed the roles of Gut1p (glycerol kinase) and Gup1p in the ability to 
accumulate radioactivity provided as $\left[{ }^{14} \mathrm{C}\right]$ glycerol against a concentration gradient, after growing the cells under derepressing conditions (Fig. 6). Deletion of GUP1 in the W303-1A genetic background clearly reduces the in/out ratio along incubation time, without reaching a plateau, supporting the notion that this strain has a reduced ability to actively take up glycerol. Furthermore, in this case the addition of the protonophore CCCP did not cause loss of radioactivity, consistently with the interpretation that glycerol kinase has converted the radioactivity from the form of glycerol to other chemical forms. This drug also did not allow incorporation of radioactivity into the cells, consistently with its long-term general inhibitory effect on metabolism, affecting the ATP level and intracellular enzymes, in particular glycerol kinase. On the other hand, the effect of deletion of GUT1 allows the establishment of the distribution of free radiolabelled glycerol in accordance with active uptake alone, i.e., without the involvement of metabolism. In this case, addition of CCCP causes a rapid relaxation of the in/out ratio to unity, as expected for an active transport system using the proton gradient.

\section{Involvement of Guplp and its close homologue in active transport in glucose-grown cells}

As mentioned above, all the selected mutants, besides being defective in growth on glycerol, were also deficient in glycerol-mediated recovery from salt stress in glucose medium. To establish this further, we investigated glycerol uptake in cells grown on glucose supplemented with $1 \mathrm{M} \mathrm{NaCl}$ and $15 \mathrm{mM}$ glycerol, as well as in cells grown on glucose alone as a control (Table 4). All strains presented in Table 4 resumed growth after widely different lag phases upon exposure to the glucose-salt-glycerol medium. Recovery from the lag was reproducible and found not to be due to selection of spontaneous mutants, but we did not investigate whether glycerol synthesis via dihydroxyacetone could be an explanation. In accordance with earlier studies (Lages and Lucas, 1997; Sutherland et al., 1997), no glycerol transport was detected in cells growing in non-stressing, glucose-based medium. The same absence was verified in cells grown under osmotic stress, but harbouring a functional glycerol synthesis pathway. Importantly, however, cells deleted in GPD1 or both $G P D 1$ and GPD2, presented glycerol uptake when growing on glucose in the presence of salt stress (Table 4). Kinetic parameters measured in this last strain under these conditions revealed the same $\mathrm{K}_{\mathrm{m}}$ but a higher $\mathrm{V}_{\max }$ ( 3 to 4 times) than in derepressed wild-type cells (compare Tables 4 and 2). The original transposon-tagged mutant, BHY22 (gpd1 $\operatorname{gpd} 2 \Delta$ gup $1 \Delta$ ) grows very poorly on 
glucose supplemented with $1 \mathrm{M} \mathrm{NaCl}$ and $10 \mathrm{mM}$ glycerol (Fig. 1). In this strain, $\mathrm{V}_{\max }$ was $60 \%$ of the value measured in gpdl gpd2 alone (Table 4). Interestingly, when we combined the gpdl and gupl mutations with a deletion of the GUP1 homologue, YPL189w, no transport is measured (Table 4), indicating that this gene is responsible for the residual uptake, present after deleting GUPI, under these growth conditions. The Ypl189wp-mediated transport system presents a $\mathrm{K}_{\mathrm{m}}$ very similar to that of Gup1p, $1.3 \mathrm{mM}$.

Because glycerol kinase has an important role in the apparent kinetics of glycerol uptake into cells grown on ethanol, we considered the possibility that it might also have a role in the glucose-grown, salt-stressed cells. Although this was, a priori, unlikely since the glycerol kinase level is low in glucose-grown cells (Sprague and Cronan, 1977), we nevertheless measured glycerol kinase activity in all strains listed in Table 4 grown on glucose and on glucose with salt and glycerol. No salt induction was seen, and all values were low, less than $10 \%$ of that seen in the wild type grown on ethanol (data not shown). We conclude that glycerol kinase is unimportant for glycerol uptake in glucose-grown, salt-stressed cells.

A glycerol transport system of the proton symport type should, as described earlier (Lages and Lucas, 1997), display proton uptake upon glycerol addition. Strains presenting glycerol uptake when exposed to salt stress also displayed significant glycerol-dependent proton uptake, while the wild type (W303-1A) did not, indicating that both Gup1p and Yp1189wp can correspond to such type of active transport system. According to (1) the molecular homology between these two genes, and (2) the apparent similarity of behaviour, as far as transport is concerned, we propose the YPL189w gene to be named GUP2.

The experiment presented in Table 4 shows that GUP1-dependent and GUP2-dependent glycerol uptake in glucose-grown cells is tightly controlled and requires the extreme conditions of the rather strong osmotic stress in $1 \mathrm{M} \mathrm{NaCl}$ combined with a deficiency in glycerol synthesis. When the GPD2 gene was deleted in a strain already partially deficient in glycerol synthesis because of a gpd1 deletion, the GUP2-dependent glycerol uptake went up by a factor of two (Table 4 ), indicating an extension of the same kind of control. On the other hand, the differences between gup1 and gup2 phenotypes suggest the two genes to be differently regulated. In this connection it may be noted that we found that Gup1p is necessary to shorten lag phase in salt-stressed cells growing on glucose: A gup1 mutant has a lag phase 2.5 times longer than the corresponding GUP1 strain, whether the latter be GPD1 GPD2 (W303-1A) or gpd1 gpd2 (data not shown). Whether the appearance of glycerol uptake in the salt-stressed cells caused by defective glycerol synthesis is due 
to an enhancement of the already high osmotic stress, or rather a direct controlling effect of low intracellular glycerol concentration, or perhaps more indirect mechanisms, remains to be investigated.

\section{Localisation of Guplp}

A gene fusion encoding a hemagglutinin-tagged version of Gup1p was constructed in order to determine cellular location(s) of the protein. The codons for the hemagglutinin (HA)-tag were inserted into an introduced restriction site five codons upstream of the stop codon, and the construct was verified by sequencing. The construct was made so that Gup1p-HA is under the control of its own promoter on a centromere-based plasmid. The construct was able to complement the growth defect of gup 1 on glycerol-based media (data not shown), indicating that at least enough of the tagged protein had the location needed for function. On a Western blot, Gup1p-HA appears as a double band at $\sim 45 \mathrm{kDa}$. This is lower than expected from the sequence $(65.3 \mathrm{kDa}$; Saccharomyces Genome Database, Stanford). A higher electrophoretic mobility for hydrophobic membrane proteins is, however, not uncommon since they bind excessive amounts of SDS (Pinson et al.,1996).

HA-Gup1p was next expressed in a strain (W3124) in which the genes encoding the three major vacuolar proteases; proteinase A, proteinase B and carboxypeptidase $\mathrm{Y}$ have been deleted to minimise unwanted proteolysis. The co-localisation of the tagged Gup1p with known marker enzymes for cellular organelles in a sucrose gradient was subsequently investigated. The cells were grown to mid-exponential phase, broken with glass beads and the membrane fraction loaded onto a continuous sucrose gradient (20\% - 55\%). Thirty fractions were collected and the enzymatic activities of known marker enzymes determined (Fig. 7). The plasma membrane was detected by measuring $\mathrm{H}^{+}$-ATPase activity, the endoplasmic reticulum by assay of NADPH-cytochrome c reductase, mitochondria by monitoring cytochrome c oxidase activity and vacuolar membranes by measuring $\alpha$-mannosidase (Villalba et al., 1992). The distribution of Gup1p was determined by Western blotting and subsequent quantification of the bands (ImageMaster, Amersham Pharmacia). Guplp peaks between 40 and $45 \%$, which corresponds to the second major peak of the plasma membrane. A minor Guplp peak is also present at $~ 38 \%$; this could correspond to the first plasma membrane peak; however, it might also represent an endoplasmic reticulum (ER) or mitochondrial 
localization (Fig. 7). 


\section{Discussion}

Saccharomyces cerevisiae has earlier been found to possess the ability to take up glycerol from the surroundings against a concentration gradient (Lages and Lucas, 1997). The uptake was shown to be driven by electrogenic proton symport. Here we describe the identification and characterisation of an open reading frame, $Y G L 084 c$, which we propose to encode a protein, Gup1p, involved in active uptake of glycerol. A homologous gene, YPL189w, GUP2, was found to have the same function under extreme conditions.

Using a mTn-lacZ/LEU2-mutagenised library (Ross-Macdonald et al., 1995), we isolated mutants defective in either utilisation of glycerol as carbon source and/or in glycerol-mediated recovery from salt stress as determined in the absence of glycerol production. Of the four different loci found to be hit in the mutants satisfying both screens, only $S P T 7$ has earlier been assigned a function. SPT7 belongs to a group of genes known to be affected in transcription start site selection (Gansheroff et al., 1995). One of the phenotypes of these mutants is slower growth on several carbon sources, including glycerol. The three other ORF's all encode putative proteins with unknown function. One of the ORF's, YGL084c, has been suggested to encode a multi-membranespanning protein belonging to the major facilitator superfamily (Nelissen et al., 1997). We call the gene GUP1, for Glycerol UPtake. From the deduced amino acid sequence, 8-10 transmembrane domains can be predicted, very similarly to the close homologue of GUP1, YPL189w.

The predicted transmembrane nature of Gup1p and Ypl189wp, together with the phenotypes of the isolated transposon mutants, made these ORF's obvious candidates for further studies, in particular the one from GUP1. The ability of GUP1 to complement the phenotypes of the transposon mutant (Fig. 3) was verified by cloning of a wild-type copy of GUP1 by GAP-repair. In an otherwise wild-type background, deletion of GUP1 results in slow growth on glycerol as sole carbon source. However, the gupl mutant also has a moderate growth defect on rich media with glucose as carbon source when supplemented with $1 \mathrm{M} \mathrm{NaCl}$, which may be interpreted to mean that even when glycerol production is not affected, salt stress requires reuptake of glycerol, leaking out of the cells. On the other hand, active uptake of glycerol under these conditions was only detected in strains with a full or partial block in glycerol synthesis.

An integral membrane localization of Gup1p is consistent with a direct role in the active uptake of glycerol. A further prediction of this role would be localisation of at least some of the molecules to the plasma membrane. We therefore separated membranes on a sucrose gradient. HA- 
tagged Gup1p was found in two peaks that coincided with the fractions containing plasma membrane. We considered the possibility that the minor one of the two Guplp peaks could represent either the ER membrane or the mitochondrial membrane. However, as both these organelles also are present at other sucrose concentrations with no corresponding peak in the Guplp profile, we find these localisations unlikely. We interpret the fact that the Gup1p profile does not coincide completely with the plasma membrane, in the beginning and the end of its curve, to be due to difficulties in quantification of the Western blotting.

The high degree of similarity between Gup1p and its close homologue ( $57 \%$ identity and $\sim 77 \%$ similarity), encoded by YPL189w, suggested that the latter might fulfil a very similar function. However, when colony growth was assayed, neither deletion nor high-copy expression of YPL189w had any effect. Further, no complementation was observed in experiments where YPL189w was expressed from a high-copy vector in a strain lacking GUP1. Over-expression with a strong promoter was not investigated. $\beta$-galactosidase assays of promoter fusions to lac $Z$ indicate that both promoters are weak, with the YPL189w promoter being around ten fold weaker than the GUP1 promoter (data not shown). Importantly, however, YPL189w was necessary for a residual active glycerol uptake in salt-stressed gpdl gupl cells on glucose. We therefore named this gene GUP2 as mentioned above.

Previous kinetic studies of cells not repressed by glucose have shown glycerol uptake to fit the sum of one diffusion component and one active saturable system (Lages and Lucas, 1997; Sutherland et al., 1997). Our uptake kinetics for the wild type is very similar to these data (Table 2). However, in the gupl mutant, which is severely impaired in its growth with glycerol as carbon source, we still found kinetics of glycerol uptake with both a diffusion component and a saturable component, although we observed changes in $\mathrm{V}_{\max }$. It is noteworthy, however, that electrogenic proton symport could no longer be measured in any of the gupl mutants, and that the addition of the protonophore CCCP did not totally prevent the residual uptake. We reasoned that the residual, GUP1-independent, uptake was probably not active as such but pulled by the metabolic trapping of incoming glycerol by glycerol kinase, encoded by GUT1, influencing the apparent uptake kinetics. This was analysed further by comparing various combinations of genes including gut 1 and gupl as well as controls. Deletion of gut 1 either alone or in combination with gpdl, only leads to smaller changes in $\mathrm{V}_{\max }$. However, when the effect of gupl was studied in a gutl gpdl background, there was a dramatic change in the kinetics of glycerol uptake (Table 2; Fig. 5). In this background, defective in glycerol synthesis and glycerol utilisation, the diffusion constant was unaffected, 
whereas the uptake with saturable kinetics was eliminated by deletion of GUP1.

Another way to analyse the components of the uptake of glycerol is to measure glycerol accumulation against a concentration gradient. The in/out accumulation ratios in Fig. 6 show that the overall uptake of glycerol consists of both active uptake by Gup1p and metabolic trapping through the action of the kinase, Gut1p. Probably because of the time course of these experiments, the catabolism has a major influence. Thus, the major part of the accumulation over time corresponds to conversion of glycerol in metabolism, which fits with the lack of efflux when CCCP is added. Moreover, in a gut 1 strain background, Gup1p was responsible for most of the overall rate of accumulation. Consistently, all of the accumulated glycerol diffused out upon addition of CCCP.

Both the uptake and accumulation results show that under derepressing conditions the glycerol uptake is the sum of a saturable, active uptake, and diffusion, and that the latter is strongly influenced by glycerol kinase, giving rise to an apparently saturable component in gupl strains. A sufficiently short uptake experiment might, in principle, overcome the kinetic artefact caused by glycerol kinase. However, in contrast to the situation with glucose, the uptake of which can be measured during less than 0.2 seconds (Walsh et al., 1994), rates of glycerol uptake are insufficient for such short uptake experiments. It is also conceivable that uptake kinetics is complicated by direct interactions between proteins involved in glycerol transport and glycerol metabolism, like the situation in Escherichia coli (Voegele et al., 1993), but we have no strong evidence for such interactions. Nevertheless, measurements of the kinase activity in ethanol-growing cells indicate that full kinase activity might be connected with functional Guplp. We can thus not exclude the possibility that there is some kind of direct interaction between Gup1p and Gut1p, affecting the activity of one or both. Despite the importance of the glycerol kinase when accumulation of radioactivity from glycerol is measured, it is clear that diffusion coupled to the metabolism is not sufficient for the cells to grow properly on glycerol as carbon and energy source, implying the physiological importance of active uptake through Gup1p.

Thus, here we describe the identification of the gene GUP1, which is essential for proper growth on glycerol, as well as for glycerol-mediated recovery from salt stress. GUP1 probably encodes a multi-membrane-spanning protein belonging to the major facilitator superfamily (Nelissen et al., 1997). By separating membranes on a sucrose gradient, we have shown that Gup1p is very likely to be present at least partly in the plasma membrane fraction.

As has earlier been seen for wild type cells (Lages and Lucas, 1997; Sutherland et al., 1997), we did not see any active glycerol uptake in any of the investigated strains when the cells 
were grown on glucose. However, when we analysed cells growing on glucose supplemented with 1 $\mathrm{M} \mathrm{NaCl}$ and $15 \mathrm{mM}$ glycerol, strains that are deficient in glycerol synthesis (gpdl or gpdl gpd2 mutants), have a strong capacity to actively transport glycerol into the cells. Although the gup 1 mutation, in this genetic background, has a clear growth phenotype, it still allows some active uptake of glycerol, due to the function of GUP2.

Considering the following: (1) in ethanol cells only Gup1p is active, (2) GUP1 is essential for growth on glycerol as sole carbon and energy source, (3) a gupl deletion increases lag phase duration of salt-stressed cells on glucose, (4) Gup2p activity is only apparent in salt-stressed cells on glucose, and (5) a gup2 deletion appears not to affect growth any carbon source, either glucose or non-fermentable ones, these two genes must be differently regulated, at least to some extent. It is noteworthy that there are two differentially controlled isoenzymes of the first step of glycerol production (Eriksson et al., 1995; Ansell et al., 1997), two differentially controlled isoenzymes of the second step of glycerol production (Norbeck et al., 1996) and now also two putative transporters of glycerol that are controlled in different ways, yet to be characterised.

In conclusion, we have found that under different metabolic conditions Gup1p and/or Gup2p are necessary for active uptake of glycerol, taking place by proton symport. We can not exclude the possibility that Gup1p and Gup2p have sensing or other regulatory functions, but the most straightforward hypothesis is that they are transporters of glycerol. This is supported by the fact that they belong to the major facilitator superfamily and by our subcellular fractionation being consistent with a plasma membrane localization.

\section{Experimental procedures}

\section{Strains and media}

The $S$. cerevisiae strains used in this study are listed in Table 5. BHY13, 21, 22, 23 and 24 were isolated as described below by transposon-based mutagenesis. BHY20-8C, 26-5D, 27-3B, 28-8C and 29-2C were isolated as spores after crossing of BHY13, 21, 22, 23 and 24, respectively, with UTL-7A. Strains with a deletion of either the GUT1 or the GUT2 gene were constructed by the twostep loop-in/loop-out technique (Rothstein, 1991). For constructing the gutl deletion, plasmid pCL370 was digested with MluI before introduction into W303-1A. CLy1 and CLy2, two 
independent isolates from the same loop-in, are isogenic except for gut1 in CLy1 and GUT1 in CLy2. Similarly, for the gut2 deletion, pBH2038 was digested with HpaI before introduction into W303-1A. BHY39 and BHY40, are two independent isolates from the same loop-in, being isogenic except for gut2 in BHY40 and GUT2 in BHY39. BHY51 was isolated after introduction and homologous recombination in YSH481 of a PCR-generated fragment containing the gene for geneticin resistance flanked by GUT1 promoter sequences and GUT1 downstream sequences (Wach et al., 1994; Wach, 1996). The desired disruption of the GUT1 gene was verified by PCR. Strains lacking YGL084c (GUP1) were constructed as follows: After integrative transformation of W3031A, YSH481, W303-1B and BHY51 with a BsaI-XhoI fragment from plasmid pBH2340, containing the his $5^{+}$gene from Schizosaccharomyces pombe flanked by YGL084c-flanking sequences, BHY54, BHY56, BHY58 and BHY66 were selected on SC plates lacking histidine. The desired deletion was verified by PCR. BHY52 was isolated after integration in strain W303-1A of a SalI-XbaI fragment from plasmid fps1 1 ::HIS3-long (obtained from M. Tamás), in which $2.3 \mathrm{~kb}$ of the coding region of FPS 1 has been replaced by the HIS3 gene. To isolate a fps $1 \Delta$ gup $1 \Delta$ double mutant, BHY52 and BHY58 were crossed and tetrads were dissected. Using PCR, BHY61-1A was found to lack both genes. Similarly, a gut $1 \Delta$ gup $1 \Delta$ double mutant was made by crossing BHY61-

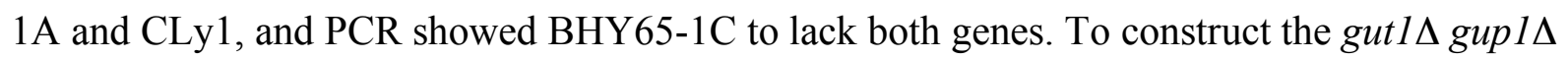

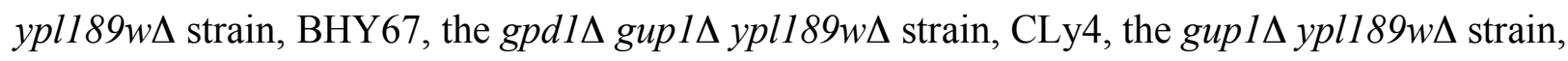
CLy3 and the ypl189w $\Delta$ mutant, CLy5, YPL189w was deleted by transformation and selection for recombinants on geneticin, using a BstBI-BsrBI fragment from pCL448 in strains BHY65-1C, BHY56, BHY54 and W303-1A, respectively. Desired substitution was confirmed by PCR.

Batch cultures of yeast were grown aerobically at $30^{\circ} \mathrm{C}$ in standard complex or defined media (Rose et al., 1990). For sporulation, pre-sporulation medium containing $0.8 \%$ yeast extract, $0.3 \%$ peptone and $10 \%$ glucose, and sporulation medium, $1 \%$ potassium acetate, $0.1 \%$ yeast extract and $0.05 \%$ glucose, were used. The inability to utilise glycerol as carbon and energy source ( $g u t$ phenotype) was recognised on glycerol-based defined media supplemented with $0.05 \%$ peptone (gut-diagnostic medium, modified from Rønnow and Kielland-Brandt, 1993). For transport assays, cells were cultivated in yeast extract-peptone-based media (Rose et al., 1990) with $2 \%$ (w/v) of ethanol or glucose as carbon sources.

Growth experiments on different plates were made as follows: Cells were inoculated in liquid medium with glucose carbon source. After dilution to $\mathrm{OD}_{600} \sim 1$, different dilutions were spotted on plates as indicated. The ability to grow was read after inoculation in $30^{\circ} \mathrm{C}$ for different 
times as indicated. Osmo-tolerance were investigated as described above using plates containing increasing concentration of $\mathrm{NaCl}$.

E. coli DH5 $\alpha$ (Sambrook et al., 1989) was used for plasmid selection and propagation and grown on LB medium according to Sambrook et al. (1989). When needed for plasmid selection and maintenance, ampicillin or kanamycin was present at $100 \mu \mathrm{g} / \mathrm{ml}$.

\section{Transposon-based mutagenesis}

Library propagation, transformation and isolation of recombinants were essentially carried out as described by Ross-Macdonald et al. (1995) and http://ycmi.med.yale.edu/YGAC/insertion_libraries.html\#mTn-lacZ/LEU2_library. From screening of around 10,000 colonies, 5 mutants were recognised by their inability to grow when replica plated onto glycerol-based ( $g u t$-diagnostic) medium and, in parallel, their inability to be rescued by 10 $\mathrm{mM}$ glycerol when exposed to $1 \mathrm{M} \mathrm{NaCl}$ on YP-based medium with $2 \%$ glucose as carbon source. All 5 mutants turned out to satisfy both criteria. Putative mutants were re-screened. To avoid mutants with generally impaired growth, and mutants defective in respiration, all mutants were also analysed for proper growth on YP-based medium with 2\% ethanol as carbon and energy source. In order to identify the genes hit by the transposon, we first had to free the isolated mutants from the URA3 gene. They were therefore crossed to yeast strain UTL-7A, and tetrads were dissected. Spores with the original mutant phenotypes and the LEU2 marker but without the URA3 marker were isolated and used as the source for rescue of the sequence flanking the transposon according to Ross-Macdonald et al. (1995).

\section{Molecular cloning and plasmid construction}

A chromosomal copy of YGL084c was cloned by a combination of PCR and homologous recombination/GAP-repair: A $1.6 \mathrm{~kb}$ fragment, covering from position -1560 to +38 , relative to the start codeine, was amplified by PCR, introducing a BamHI site in the downstream end. The fragment was blunt-end inserted into the EcoRV site in pBC sk+ (Stratagene), yielding pBH2100. Similarly, a 824 bp fragment, covering from position +1792 to +2515 , relative to the start codeine, 
was amplified and blunt-end inserted in the EcoRV site in pBC sk + (Stratagene), yielding pBH2132. Recloning of an NsiI-BamHI fragment from pBH2100 between the PstI and BamHI sites of pRS314 (Sikorski and Hieter, 1989) created pBH2128. After cutting pBH2132 with BstEII, filling in the 5'-overhang and digestion with $B a m H I$, the resulting $\sim 285 \mathrm{bp}$ fragment was inserted into pRS316 (Sikorski and Hieter, 1989), which had previously been digested with XbaI and subjected to filling in of the 5'-overhangs and cleavage with BamHI. This resulted in pBH2138. pBH2166 was constructed by recloning of a KpnI-BamHI fragment from pBH2128 between the same sites in pBH2138. pBH2178, containing the gap-repaired version of YGLO84c along with its promoter, was made as follows: pBH2166 was digested with BamHI and introduced into yeast strain W303-1A, selecting for URA3. After isolation from yeast cells, the plasmid was propagated in E. coli. Recloning of a KpnI-SacI fragment from pBH2178 between the corresponding sites in pRS306 (Sikorski and Hieter, 1989) and pRS426 (Christianson et al., 1992) resulted in pBH2188 and pBH2198, respectively. pBH2200 was constructed after digestion of pBH2188 with HpaI and SpeI, the 5'overhang was filled in and the linearised plasmid was subsequently re-ligated. pBH2209 was constructed by insertion of a $1 \mathrm{~kb}$ BamHI fragment from pBH2100 into the BamHI site in plasmid pFN8 (Nagawa and Fink, 1985). The entire promoter region of $Y G L 084 c$, fused to lacZ in pBH2209 was sequenced and found to be identical to the already published sequence (Saccharomyces Genome Database, Stanford). pBH2340 was constructed as follows: A BglIIEcoRV fragment from pFA6a-GFPMT-HIS3MX6 (Wach et al., 1997), containing the his $5^{+}$gene from S. pombe, was inserted into BglII- and HpaI-digested pBH2188, replacing the whole YGL084c coding region with non-Saccharomyces DNA. A 574 bp fragment of sequences downstream of $Y G L 084 c$ was amplified by PCR, introducing an NcoI site just downstream of YGL084c. The obtained fragment was inserted into SmaI-digested pRS306 (Sikorski and Hieter, 1989), to give pBH2310. YGL084c was inserted to be under the control of the TPI promoter in plasmid pYX212 (R\&D systems): pBH2294 was digested with AatII and BamHI and inserted between the corresponding sites in pYX212, resulting in pBH2320. pBH2320 was subsequently digested with EcoRI, sticky ends were filled in using Klenow, and following digestion with AflII, a HpaI-AflII fragment from pBH2178 was inserted, yielding pBH2330. HA-tagging of YGL084c was carried out by cloning of a NcoI-ClaI fragment from pBH2310, where the sticky end of the NcoI site had been filled in by Klenow treatment, between the ClaI site and the SmaI site of pBH2330, resulting in pBH2344. This plasmid is thus derived from the $2 \mu$-based vector pYX212 and carries YGL084c behind the strong TPI promoter. A $2 \mu$-based plasmid carrying the HA-tagged YGL084c behind its 
own promoter was also generated; pCL577 was made by inserting a 1080 bp AflII-SacI fragment from $\mathrm{pBH} 2344$ between the corresponding sites of pBH2178. To generate pCL583, pCL577 was digested with $K p n I$ and $S a c I$ and inserted between the corresponding sites in pRS316 (Sikorski and Hieter, 1989). This construct contains YGL084c-HA on a centromere-based plasmid.

A chromosomal copy of YPL189w was cloned by a combination of PCR and GAP-repair: A $2375 \mathrm{bp}$ fragment, covering from position -2323 to +52 , relative to the start codeine, was amplified by PCR, introducing a BamHI site in the downstream end of the fragment. The fragment was digested with HindIII and BamHI and inserted between the corresponding sites of pFN8 (Nagawa and Fink, 1985), resulting in plasmid pBH2262. The entire promoter region of YPL189w fused to lacZ in pBH2262 was sequenced and found to be identical to the already published sequence. A 829 bp BamHI-HindIII fragment from pBH2262 was subsequently inserted into the corresponding sites in pRS316 (Sikorski and Hieter, 1989), yielding pCL409. A 600 bp fragment covering from position +1990 to +2589 , relative to the start codeine, was amplified by PCR. The fragment was blunt-end-inserted into the EcoRV site in pBC sk+ (Stratagene), giving pBH2097. pCL439 was made by insertion of a BamHI-HincII fragment from pBH2097 into EcoICRI and BamHI-digested pCL409. pCL478, containing the gap-repaired copy of YPL189w along with its promoter, was made as follows: pCL439 was digested with BamHI and BstXI and introduced into yeast strain W303-1A, selecting for URA3. After recovery from yeast, the plasmid was propagated in E. coli. pCL448 was constructed as follows: A 810 bp PvuII-BamHI fragment from pBH2262 was inserted into SmaIBglII-digested pFA6-KanMX3 (Wach et al., 1994). The resulting plasmid, pCL430, was digested with EcoRI and a 527 bp EcoRI fragment from pBH2097 was inserted, giving pCL448. A highcopy plasmid containing YPL189w was made as follows: A $3.4 \mathrm{~kb}$ ClaI fragment from pCL478 was inserted into the ClaI site of pRS426 (Christianson et al., 1992), giving pCL489. pGut2-2 (Rønnow and Kielland-Brandt, 1993) was digested with SnaBI and re-ligated, resulting in pBH2020. pBH2020 was subsequently digested with $P v u I I$ and BamHI and inserted between the SmaI and BamHI sites of pRS306 (Sikorski and Hieter, 1989) to give pBH2038. A 819 bp HindIII-SpeI fragment from pGut1-6 (Rønnow, 1992) was inserted between the HindIII and SpeI sites of pRS306 (Sikorski and Hieter, 1989), and the resulting plasmid was digested with SpeI and NotI and the latter site was filled in using Klenow. Then a 1453 bp PvuII-SpeI fragment from pGut1-6 (Rønnow, 1992) was inserted into the linearised plasmid. This resulted in pCL370. 


\section{Kinetics of glycerol uptake}

Cells were grown in complex medium and harvested in mid-exponential phase, regardless of the carbon source or the presence of salt stress, by centrifugation, washed twice and re-suspended to a final concentration of $\sim 30 \mathrm{mg}$ dry wt. $\mathrm{ml}^{-1}$ in ice-cold distilled water. In the case of salt-grown cells, washing, re-suspension and assays were carried out at the same $\mathrm{NaCl}$ concentrations. Initial uptake rates of glycerol were determined according to the methodologies described before (Lages and Lucas, 1997), using $\left[{ }^{14} \mathrm{C}\right]$ glycerol solutions with variable specific activity, ranging from 0.2 to $50 \mathrm{mM}$ with 2800 to $250 \mathrm{dpm} / \mathrm{nmol}$, respectively. Computer regression analysis program GraphPad PRISM $^{\mathrm{R}}$ (GraphPad Software, Inc.) was used to calculate kinetic parameters.

Glycerol accumulation ratios were determined as described by Lages and Lucas (1997). The efflux of radiolabelled glycerol was assayed with $50 \mu \mathrm{M}$ CCCP (carbonyl cyanide $m$-chlorophenyl hydrazone), a protonophore which was also used for control, as an inhibitor to prevent accumulation.

Glycerol-induced proton uptake was measured as described by Lages and Lucas (1997). Cells were grown in glucose-based complex medium (YPD) supplemented with $1 \mathrm{M} \mathrm{NaCl}$ and 15 $\mathrm{mM}$ glycerol at $30^{\circ} \mathrm{C}$. Initial uptake rates of protons were calculated as the change of the tangential slope of the alkalinisation curve, immediately after addition of $10 \mathrm{mM}$ glycerol to the cell suspension, and normalised to cell dry weight. Calibration of $\mathrm{pH}$ variations was performed using 10 $\mu 110.0 \mathrm{mM} \mathrm{HCl}$.

\section{Glycerol kinase assays}

Glycerol kinase activity in cell-free extracts was determined according to Krakow and Wang (1990). This method is based on glycerol 3P-dehydrogenase (Boehringer Mannheim 127752)mediated glycerol 3P oxidation. $\mathrm{NAD}^{+}$reduction was followed at $340 \mathrm{~nm}$. The reaction mixture was composed of $667 \mu 1$ buffer ( $0.3 \mathrm{M}$ glycine, $0.7 \mathrm{M}$ hydrazine, $\mathrm{pH} 9.0), 33 \mu \mathrm{NAD}, 33 \mu 1$ glycerol (0.3M), $33 \mu 1 \mathrm{MgCl}_{2}(0.15 \mathrm{M}), 10$ to $20 \mu \mathrm{l}$ cell extract and ultra-pure water to $1 \mathrm{ml}$ volume. ATP $(0.15 \mathrm{M})$ and glycerol were alternatively used as starters. Cell-free extracts were prepared by centrifuging and washing twice $250 \mathrm{ml}$ cell culture at $7000 \mathrm{rpm}, 2 \mathrm{~min}$ at $4^{\circ} \mathrm{C}$. Pellets were either used directly or kept frozen at $-70^{\circ} \mathrm{C}$. Prior to utilisation, these were incubated in buffer $(50 \mathrm{mM}$ 
MES, $1 \mathrm{mM}$ EDTA, $150 \mathrm{mM}\left(\mathrm{NH}_{4}\right)_{2} \mathrm{SO}_{4}, \mathrm{NaOH}$ to $\mathrm{pH} 6.0$ and freshly prepared DTT to $1 \mathrm{mM}$, broken in a bead mill (1.5 g $0.5 \mathrm{~mm} \varnothing$ glass beads) for $4 \times 1 \mathrm{~min}$, intercalated with $1 \mathrm{~min}$ rest on ice, followed by centrifugation $\left(1500 \mathrm{~g}, 15 \mathrm{~min}\right.$, at $4^{\circ} \mathrm{C}$ ). Complete ${ }^{\mathrm{TM}}$ Boehringer Mannheim protease inhibitor (CAT\# 1697498) was added. Protein content in extracts was quantified using FolinCiocalteau reagent.

\section{Subcellular fractionation}

Strain W3124 (prb1 pep4 prc1) transformed with pCL583 was grown in a glucose-based complete medium with glutamate as nitrogen source to late exponential phase $\left(\mathrm{OD}_{600} \sim 2\right)$, and the cells were broken with glass beads as described (Villalba et al., 1992). Cell debris was removed by 15 min centrifugation at $700 \mathrm{~g}$ and total membranes were collected by centrifuging the supernatant for 60 min at 40,000 rpm (Beckman 60 Ti rotor). Sucrose gradient fractionation of membranes and determination of marker enzyme distribution were carried out according to Villalba et al. (1992). Gup1p distribution was detected with Western blotting and ECL+Plus chemiluminescence according to the manufacturer (Amersham Pharmacia). The primary antibody was high-affinity rat monoclonal antibody directed against the HA-tag (Boehringer Mannheim) and the secondary antibody was peroxidase-coupled anti-rat IgG (Amersham Pharmacia). 


\section{References}

Albertyn, J., Hohmann, S., Thevelein, J.M., and Prior, B.A. (1994) GPD1, which encodes glycerol3-phosphate dehydrogenase, is essential for growth under osmotic stress in Saccharomyces cerevisiae, and its expression is regulated by the high-osmolarity glycerol response pathway. Mol Cell Biol 14: 4135-4144.

Ansell, R., Granath, K., Hohmann, S., Thevelein, J.M., and Adler, L. (1997) The two isoenzymes for yeast $\mathrm{NAD}^{+}$-dependent glycerol 3-phosphate dehydrogenase encoded by GPD1 and GPD2 have distinct roles in osmoadaptation and redox regulation. EMBO J 16: 2179-2187.

Blomberg, A. (1997) Osmoresponsive proteins and functional assessment strategies in Saccharomyces cerevisiae. Electrophoresis 18: 1429-1440.

Christianson, T.W., Sikorski, R.S., Dante, M., Shero, J.H., and Hieter, P. (1992) Multifunctional yeast high-copy-number shuttle vectors. Gene 110: 119-122.

Eriksson, P., André, L., Ansell, R., Blomberg, A., and Adler, L. (1995) Cloning and characterization of GPD2, a second gene encoding sn-glycerol 3-phosphate dehydrogenase $\left(\mathrm{NAD}^{+}\right)$in Saccharomyces cerevisiae, and its comparison with GPD1. Mol Microbiol 17: 95107.

Gancedo, C., Llobell, A., Ribas, J.-C., and Luchi, F. (1986) Isolation and characterization of mutants from Schyzosaccharomyces pombe defective in glycerol catabolism. Eur J Biochem 159: $171-174$.

Gansheroff, L.J., Dollard, C., Tan, P., and Winston, F. (1995) The Saccharomyces cerevisiae SPT7 gene encodes a very acidic protein important for transcription in vivo. Genetics 139: 523-536.

Kyte, J., and Doolittle, R.F. (1982) A simple method for displaying the hydropathic character of a protein. J Mol Biol 157: 105-132.

Krakow, J.L., and Wang, C.C. (1990) Purification and characterization of glycerol kinase from Trypanosoma brucei. Mol Biochem Parasitol 43: 17-26.

Lages, F., and Lucas, C. (1997) Contribution to the physiological characterization of glycerol active uptake in Saccharomyces cerevisiae. Biochim Biophys Acta 1322: 8-18.

Larsson, K., Ansell, R., Eriksson, P., and Adler, L. (1993) A gene encoding sn-glycerol 3-phosphate dehydrogenase $\left(\mathrm{NAD}^{+}\right)$complements an osmosensitive mutant of Saccharomyces cerevisiae. Mol Microbiol 10: 1101-1111. 
Luyten, K., Albertyn, J., Skibbe, W.F., Prior, B.A., Ramos, J., Thevelein, J.M., and Hohmann, S. (1995). Fps1, a yeast member of the MIP family of channel proteins, is a facilitator for glycerol uptake and efflux and is inactive under osmotic stress. EMBO J 14: 1360-1371.

May, J.W., Marshall, J.H., and Sloan, J. (1982) Glycerol utilization by Schizosaccharomyces pombe: phosphorylation of dihydroxyacetone by a specific kinase as the second step. $J$ Gen Microbiol 128: 1763-1766.

Nagawa, F., and Fink, G.R. (1985) The relationship between the "TATA" sequence and transcription initiation sites at the HIS4 gene of Saccharomyces cerevisiae. Proc Natl Acad Sci USA 82: 8557-8561.

Nelissen, B., De Wachter, R., and Goffeau, A. (1997) Classification of all putative permeases and other membrane plurispanners of the major facilitator superfamily encoded by the complete genome of Saccharomyces cerevisiae. FEMS Microbiol Rev 21: 113-134.

Norbeck, J., Påhlman, A.-K., Akhtar, N., Blomberg, A., and Adler, L. (1996) Purification and characterization of two isoenzymes of DL-Glycerol-3-phosphatase from Saccharomyces cerevisiae. Identification of the corresponding GPP1 and GPP2 genes and evidence for osmotic regulation of Gpp2p expression by the osmosensing mitogen-activated protein kinase signal transduction pathway. J Biol Chem 271: 13875-13881.

Norbeck, J., and Blomberg, A. (1997) Metabolic and regulatory changes associated with growth of Saccharomyces cerevisiae in 1.4 M NaCl. J Biol Chem 272: 5544-5554.

Orr-Weaver, T.L., and Szostak, J.W. (1983) Yeast recombination: the association between doublestrand gap repair and crossing-over. Proc Natl Acad Sci USA 80: 4417-4421.

Pavlik, P., Simon, M., Schuster, T., and Ruis, H. (1993). The glycerol kinase (GUT1) gene of Saccharomyces cerevisiae: cloning and characterization. Curr Genet 24: 21-25.

Pinson, B., Pillois, X., Brèthes, D., Chevallier, J., and Napias, C. (1996) In vivo phosphorylation of the purine/cytosine permease from the plasma membrane of the yeast Saccharomyces cerevisiae. Eur J Biochem 239: 439-444.

Rose, M.D., Winston, F., and Hieter, P. (1990) Methods in Yeast Genetics: A Laboratory Course Manual. New York: Cold Spring Harbor Laboratory Press.

Ross-Macdonald, P., Burns, N., Malczynski, M., Sheehan, A., Roeder, S., and Snyder, M. (1995) Methods for large-scale analysis of gene expression, protein localization, and disruption phenotypes in Saccharomyces cerevisiae. Methods Mol Cell Biol 5: 298-308. 
Rothstein, R. (1991) Targeting, disruption, replacement, and allele rescue: Integrative DNA transformation in yeast. Methods Enzymol 194: 281-301.

Rønnow, B., and Kielland-Brandt, M.C. (1993) GUT2, a gene for mitochondrial glycerol 3phosphate dehydrogenase of Saccharomyces cerevisiae. Yeast 9: 1121-1130.

Rønnow, B. (1992) Glycerol utilization in Saccharomyces cerevisiae. Ph.D. thesis, University of Copenhagen, November 1992.

Sambrook, J., Fritsch, E.F., and Maniatis, T. (1989) Molecular Cloning: A Laboratory Manual. Cold Spring Harbor, New York: Cold Spring Harbor Laboratory Press.

Sikorski, R.S., and Hieter, P. (1989) A system of shuttle vectors and yeast host strains designed for efficient manipulation of DNA in Saccharomyces cerevisiae. Genetics 122: 19-27.

Sprague, G.F. Jr., and Cronan J.E. Jr. (1977) Isolation and characterization of Saccharomyces cerevisiae mutants defective in glycerol catabolism. J Bacteriol 129: 1335-1342.

Sutherland F.C.W., Lages, F., Lucas, C., Luyten, K., Albertyn J., Hohmann, S., Prior, B.A., and Kilian, S.G. (1997) Characteristics of Fps1-dependent and -independent glycerol transport in Saccharomyces cerevisiae. J Bacteriol 179: 7790-7795.

Tamás, M.J., Luyten, K., Sutherland, F.C.W., Hernandez, A., Albertyn, J., Valadi, H., Li, H., Prior, B.A., Kilian, S.G., Ramos, J., Gustafsson, L., Thevelein, J.M., and Hohmann, S. (1999) Fps1p controls the accumulation and release of the compatible solute glycerol in yeast osmoregulation. Mol Microbiol 31: 1087-1104.

Thomas, B.J., and Rothstein, R. (1989) Elevated recombination rates in transcriptionally active DNA. Cell 56: 619-630.

Van Aelst, L., Hohmann, S., Zimmermann, F.K., Jans, A.W.H., and Thevelein, J.M. (1991) A yeast homologue of the bovine lens fibre MIP gene family complements the growth defect of a Saccharomyces cerevisiae mutant on fermentable sugars but not its defect in glucose-induced RAS-mediated cAMP signalling. EMBO J 10: 2095-2104.

Varela, J.C.S., and Mager, W.H. (1996) Response of Saccharomyces cerevisiae to changes in external osmolarity. Microbiology 142: 721-731.

Villalba, J.M., Palmgren, M.G., Berberián, G.E., Ferguson, C., and Serrano, R. (1992) Functional expression of plant plasma membrane $\mathrm{H}^{+}$-ATPase in yeast endoplasmic reticulum. $J$ Biol Chem 267: 12341-12349.

Voegele, R.T., Sweet, G.D., and Boos, W. (1993) Glycerol kinase of Escherichia coli is activated by interaction with the glycerol facilitator. J Bacteriol 175: 1087-1094. 
Wach, A., Brachat, A., Pöhlmann, R., and Philippsen, P. (1994) New heterologous modules for classical or PCR-based gene disruptions in Saccharomyces cerevisiae. Yeast 10: 1793-1808.

Wach, A. (1996) PCR-synthesis of marker cassettes with long flanking homology regions for gene disruptions in S. cerevisiae. Yeast 12: 259-265.

Wach, A., Brachat, A., Alberti-Segui, C., Rebischung, C., and Philippsen, P. (1997) Heterologous HIS3 marker and GFP reporter modules for PCR-targeting in Saccharomyces cerevisiae. Yeast 13: $1065-1075$.

Walsh, M.C., Smits, H.-P., and Van Dam, K. (1994) Respiratory inhibitors affect incorporation of glucose into Saccharomyces cerevisiae cells, but not the activity of glucose transport. Yeast 10: 1553-1558.

Winston, F., Dollard, C., Malone, E.A., Clare, J., Kapakos, J.G., Farabaugh, P., and Minehart, P.L. (1987) Three genes are required for trans-activation of Ty transcription in yeast. Genetics 115: 649-656.

Winston, F., Chaleff, D.T., Valent, B., and Fink, G.R. (1984) Mutations affecting Ty-mediated expression of the HIS4 gene of Saccharomyces cerevisiae. Genetics 107: 179-197. 
Table 1. Positions of transposon insertions

\begin{tabular}{lllcl}
\hline $\begin{array}{l}\text { Tn- } \\
\text { mutant } \\
\text { strain }\end{array}$ & Affected gene & $\begin{array}{l}\text { ORF length } \\
\text { (amino acids) }\end{array}$ & $\begin{array}{l}\text { Position of transposon } \\
\text { insertion }^{\mathrm{a}}\end{array}$ & $\begin{array}{l}\text { Rescue } \\
\text { strain }^{\mathrm{b}}\end{array}$ \\
\hline BHY13 & YDL074c & 700 & $1463 \mathrm{~T}$ (o.f.) & BHY20-8C \\
BHY21 & YPL180w & 799 & $2326 \mathrm{C}$ (o.f.) & BHY27-5B \\
BHY22 & GUP1 & 560 & 165 C (i.f.) & BHY29-2C \\
BHY23 & SPT7 & 1332 & 1803 A (i.f.) & BHY25 \\
BHY24 & YDL074c & 700 & 1463 T (o.f.) & BHY26-5D \\
\hline
\end{tabular}

\footnotetext{
${ }^{a}$ Transposon is inserted after indicated nucleotide. o.f. indicates an out-of-frame fusion. i.f. indicates an in-frame fusion. ${ }^{\mathrm{b}}$ LEU2 ura3 derivatives of the original transposon mutants, see Experimental procedures. A $U R A 3$-based plasmid was introduced into each rescue strain allowing rescue of part of the disrupted gene for identification.
} 
Table 2. Kinetic parameters of initial $\left[{ }^{14} \mathrm{C}\right]$ glycerol uptake at $30^{\circ} \mathrm{C}$ and $\mathrm{pH} 5.0$ in cells grown in ethanol-based complex medium (YP-ethanol)

\begin{tabular}{llll}
\hline $\begin{array}{l}\text { Relevant } \\
\text { Genotypes }\end{array}$ & $\begin{array}{l}\mathrm{K}_{\mathrm{m}} \\
(\mathrm{mM})\end{array}$ & $\begin{array}{l}\mathrm{V}_{\max } \\
\left(\mu \mathrm{mol} \cdot \mathrm{h}^{-1} \cdot \mathrm{g} \mathrm{dw}^{-1}\right)\end{array}$ & $\begin{array}{l}\mathrm{D} \\
\left(1 \cdot \mathrm{h}^{-1} \cdot \mathrm{g} \mathrm{dw}^{-1}\right)\end{array}$ \\
\hline Wild type & $1.1 \pm 0.2(3)$ & $277 \pm 26(3)$ & $0.010(1)$ \\
gup1 & $1.5 \pm 0.5(4)$ & $181 \pm 12(4)$ & $0.009(1)$ \\
gup1 fps1 & $1.4 \pm 0.6(3)$ & $137 \pm 10(3)$ & $0.008(1)$ \\
gup1 ypl189w & $1.6 \pm 0.7(3)$ & $169 \pm 17(3)$ & $0.008(1)$ \\
gut1 & $1.3 \pm 0.4(4)$ & $205 \pm 17(4)$ & $0.009(4)$ \\
gut1 gpd1 & $1.8 \pm 0.6(4)$ & $248 \pm 36(4)$ & $0.011(3)$ \\
gut1 gpd1 gup1 & $-(3)$ & $-(3)$ & $0.007(1)$ \\
gut1 gup1 ypl189w & $-(2)$ & $-(2)$ & $0.007(1)$
\end{tabular}

All strains are derived from W303-1A; only relevant genotypes are shown.

- means that no active uptake was detected.

Numbers in parenthesis indicate numbers of independent assays 
Table 3. Kinetic parameters of initial uptake rates of $\left[{ }^{14} \mathrm{C}\right]$ glycerol at $30^{\circ} \mathrm{C}$ and $\mathrm{pH} 5.0$ in cells grown in ethanol-based complex medium (YP-ethanol)

\begin{tabular}{|c|c|c|c|}
\hline $\begin{array}{l}\text { Relevant } \\
\text { genotypes }\end{array}$ & $\begin{array}{l}\mathrm{K}_{\mathrm{m}} \\
(\mathrm{mM})\end{array}$ & $\begin{array}{l}\mathrm{V}_{\max } \\
\left(\mu \mathrm{mol} \cdot \mathrm{h}^{-1} \cdot \mathrm{g} \mathrm{dw}^{-1}\right)\end{array}$ & $\begin{array}{l}\mathrm{D} \\
\left(1 \cdot \mathrm{h}^{-1} \cdot \mathrm{gdw}^{-1}\right)\end{array}$ \\
\hline Wild type & $1.1 \pm 0.2(3)$ & $277 \pm 26(3)$ & $0.010 \pm 0.001$ \\
\hline gutl & $1.3 \pm 0.4(4)$ & $205 \pm 17(4)$ & $0.009 \pm 0.001$ \\
\hline gut2 & $1.5 \pm 0.7(3)$ & $212 \pm 48(3)$ & $0.008 \pm 0.002$ \\
\hline gppl & $1.6 \pm 0.7(3)$ & $326 \pm 62(3)$ & $0.011 \pm 0.002(3)$ \\
\hline gpp2 & $1.9 \pm 0.2(2)$ & $318 \pm 20(2)$ & $0.009 \pm 0.001(2)$ \\
\hline gpp1 gpp2 & $1.5 \pm 0.3(3)$ & $250 \pm 27$ & $0.007 \pm 0.001$ \\
\hline gpdl & $1.3 \pm 0.3(2)$ & $256 \pm 27(2)$ & $0.008 \pm 0.001$ \\
\hline gpd2 & $1.2(1)$ & $252(1)$ & $0.009(1)$ \\
\hline gpd1 gpd2 & $1.6 \pm 0.4(3)$ & $237 \pm 30(3)$ & $0.013 \pm 0.001$ \\
\hline
\end{tabular}

All strains are derived from W303-1A; only relevant genotypes are shown.

Numbers in parenthesis indicate numbers of independent assays 
Table 4. Estimation of the $\mathrm{V}_{\max }\left(\mu \mathrm{mol} \cdot \mathrm{h}^{-1} \cdot \mathrm{g} \mathrm{dw}^{-1}\right)$ of glycerol uptake into salt-stressed or unstressed cells grown in glucose-based complex medium at $30^{\circ} \mathrm{C}$ and $\mathrm{pH} 5.0$ by measuring uptake of $\left[{ }^{14} \mathrm{C}\right]$ glycerol at an extracellular concentration of $2 \mathrm{mM}$

\begin{tabular}{lcc}
\hline Relevant & \multicolumn{2}{c}{ Growth medium (YPD) } \\
Genotypes & Glucose & Glucose \\
& & $+1 \mathrm{M} \mathrm{NaCl}+15 \mathrm{mM}$ Glycerol \\
\hline Wild type & $<50$ & $<50$ \\
gpd1 gpd2 & $<50$ & $630 \pm 18(3)$ \\
gup1 & $<50$ & $<50$ \\
gut1 & $<50$ & $<50$ \\
gup1 gut1 & $<50$ & $<50$ \\
gpd1 gup1 & $<50$ & $<50$ \\
gup1 ypl189w & $<50$ & $<50$ \\
gpd1 gup1 ypl189w & $<50$ & $379 \pm 37(2)$ \\
gpd1 gpd2 gupl & $<50$ & $<5(2)$ \\
\hline
\end{tabular}

All strains are derived from W303-1A; only relevant genotypes are shown.

Numbers in parenthesis indicate numbers of independent assays 
Table 5. Strains used in this study

\begin{tabular}{|c|c|c|}
\hline W303-1A & MATa leu2-3,112 ura3-1 trp1-1 his3-11,15 ade2-1 can1-100 & $\begin{array}{l}\text { Thomas and Rothstein, } \\
1989\end{array}$ \\
\hline W303-1B & Isogenic to W303-1A but MAT? & $\begin{array}{l}\text { Thomas and Rothstein, } \\
1989\end{array}$ \\
\hline W303-2n & Diploid of W303-1A and W303-1B & $\begin{array}{l}\text { Thomas and Rothstein, } \\
1989\end{array}$ \\
\hline UTL-7A & MATa ura3-52 leu2 trp1 gal2 gal10 & Heinisch, J. \\
\hline YSH481 & Isogenic to W303-1A but MATa gpd1::LEU2 & Hohmann, S. \\
\hline $\begin{array}{l}\text { YSH6.142- } \\
\text { 3D }\end{array}$ & Isogenic to W303-1A but MAT $\alpha$ gpd1::TRP1 gpd2::URA3 & Hohmann, S. \\
\hline YSH392 & Isogenic to W303-1A but gpd1::TRP1 & Hohmann, S. \\
\hline $\begin{array}{l}\text { YSH6.141- } \\
4 \mathrm{~A}\end{array}$ & Isogenic to W303-1A but $g p d 2:: U R A 3$ & Hohmann, S. \\
\hline YSH642 & $\begin{array}{l}\text { Isogenic to W303-1A but } g p d 1: \because T R P 1 \text { gpd2::URA3 } \\
\text { Isogenic to W303-1A but MAT } \operatorname{gpp} 1: \because K a n M X 4\end{array}$ & $\begin{array}{l}\text { Hohmann, S. } \\
\text { Påhlman, A.-K. and } \\
\text { Adler, L. }\end{array}$ \\
\hline & Isogenic to W303-1A but gpp $2:: K a n M X 4$ & $\begin{array}{l}\text { Påhlman, A.-K. and } \\
\text { Adler, L. }\end{array}$ \\
\hline & Isogenic to W303-1A but gpp $1: \because K a n M X 4$ gpp $2:: H I S 3 M X 6$ & $\begin{array}{l}\text { Påhlman, A.-K. and } \\
\text { Adler, L. }\end{array}$ \\
\hline W3124 & $\begin{array}{l}\text { MATa ura3-52 leu2-3,112 his3-200 prc1 }:: H I S 3 \text { prb1 }:: \text { LEU2 } \\
\text { pep4-1137 }\end{array}$ & Winther, J.R. \\
\hline BHY13 & 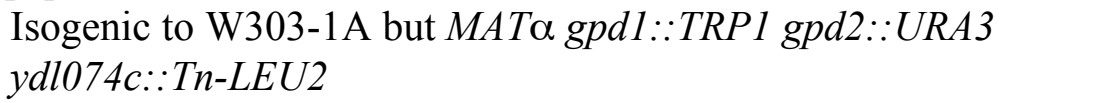 & This study \\
\hline BHY20-8C & MAT $\alpha$ ura3 leu 2 trp1 ade2 gpd1::TRP1 ydl074c::Tn-LEU2 & This study \\
\hline BHY21 & 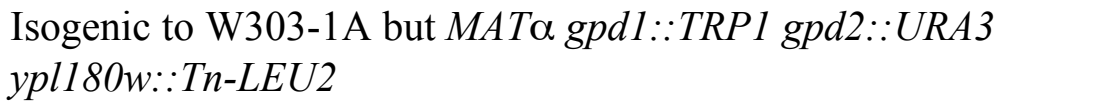 & This study \\
\hline BHY22 & 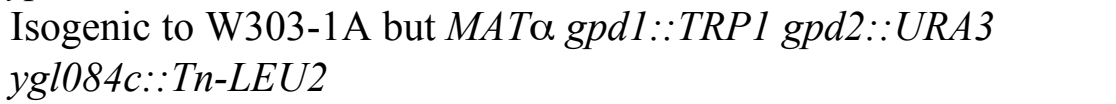 & This study \\
\hline BHY23 & $\begin{array}{l}\text { Isogenic to W303-1A but MATa gpd1::TRP1 gpd2::URA3 } \\
\text { spt7::Tn-LEU2 }\end{array}$ & This study \\
\hline BHY24 & $\begin{array}{l}\text { Isogenic to W303-1A but MATa gpd1::TRP1 gpd2::URA3 } \\
y d l 074 c:: T n-L E U 2\end{array}$ & This study \\
\hline BHY26-5D & MATa ura3 leu2 trp1 his3 ade2 gpd1::TRP1 ydl074c::Tn-LEU2 & This study \\
\hline BHY27-5B & MAT $\alpha$ ura3 leu 2 trp1 his 3 ade2 gpd1::TRP1 ypll80w::Tn-LEU2 & This study \\
\hline BHY29-2C & MATa ura3 leu2 trp1 his3 gpd1::TRP1 ygl084c::Tn-LEU2 & This study \\
\hline BHY28-8C & MATa ura3 leu2 trp1 his3 gpd1::TRP1 spt7::Tn-LEU2 & This study \\
\hline BHY39 & Isogenic to W303-1A GUT2 & This study \\
\hline BHY40 & Isogenic to W303-1A but gut2 & This study \\
\hline BHY 51 & Isogenic to W303-1A but MAT $\alpha$ gpd1::LEU2 gut1::KanMX & This study \\
\hline BHY 52 & Isogenic to W303-1A but fps $1: \because H I S 3$ & This study \\
\hline BHY54 & Isogenic to W303-1A but $y g l 084 c:: H i s 5^{+}$ & This study \\
\hline BHY56 & Isogenic to W303-1A but MAT $\alpha$ gpd1::LEU2 ygl084c::His $5^{+}$ & This study \\
\hline BHY58 & Isogenic to W303-1A but MAT $\alpha$ ygl084c::His $5^{+}$ & This study \\
\hline BHY61-1A & 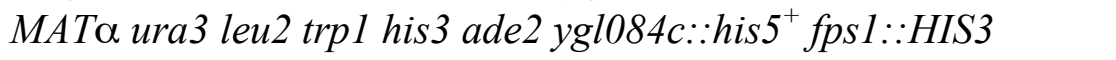 & This study \\
\hline
\end{tabular}




\begin{tabular}{|c|c|c|}
\hline BHY65-1C & MATа ura3 leu 2 trpl his 3 ade2 gut1 ygl084c:: his $5^{+}$ & This study \\
\hline BHY66 & $\begin{array}{l}\text { Isogenic to W303-1A but MAT } \alpha \text { gpd1::LEU2 gut } 1: \text { KanMX } \\
\text { ygl084c::His } 5^{+}\end{array}$ & This study \\
\hline BHY67 & $\begin{array}{l}\text { MATa } \text { ura3 leu2 trp1 his3 ade2 gut1 ygl084c::his } 5^{+} \\
\text {ypl189w:KanMX }\end{array}$ & This study \\
\hline CLy1 & Isogenic to W303-1A but gut1 & This study \\
\hline CLy2 & Isogenic to W303-1A GUT1 & This study \\
\hline CLy3 & Isogenic to W303-1A but ygl084c::His $5^{+}$ypl189w::KanMX & This study \\
\hline CLy4 & $\begin{array}{l}\text { Isogenic to W303-1A but gpd1::LEU2 ygl084c::His } 5^{+} \\
\text {ypll89w::KanMX }\end{array}$ & This study \\
\hline CLy5 & Isogenic to W303-1A but ypl189w::KanMX & This study \\
\hline
\end{tabular}




\section{Figure legends}

Fig. 1. Phenotypes of mutants isolated in a gpd1 gpd 2 strain. Cells were grown in YPD at $30^{\circ} \mathrm{C}$ to $\mathrm{OD}_{600} \sim 3$ and diluted to $\mathrm{OD}_{600}=1$. Ten $\mu 1$ of a $10^{-1}, 10^{-2}, 10^{-3}$ and $10^{-4}$ dilution were spotted on different media. Upper panel: Growth on glycerol as carbon and energy source. Lower panel: YPD plates containing $1 \mathrm{M} \mathrm{NaCl}$ and supplemented with $10 \mathrm{mM}$ glycerol. Lane 1, parental strain (YSH6.142-3D); Lane 2, BHY13; Lane 3, BHY21; Lane 4, BHY22; Lane 5, BHY23; Lane 6, BHY24. Plates were incubated at $30^{\circ} \mathrm{C}$ for $72 \mathrm{~h}$ and $48 \mathrm{~h}$, respectively.

Fig. 2. Alignment of Gup1p with the close homologue Yp1189wp (Gup2p) and Spac24h6-01cp from S. pombe using LASERGENE from DNA STAR, Inc., Madison. A, Amino acids that are conserved between at least two of the sequences are shown in closed boxes. B, Hydrophobicity profiles calculated according to Kyte and Doolittle (1982).

Fig. 3. Plasmid complementation of strain BHY29-2C, disrupted in YGL084c (GUP1) by the transposon. A, Cells were grown and spotted as in described in the legend to Figure 1. Upper panel: Growth on glycerol as carbon and energy source. Lower panel: YPD containing $1 \mathrm{M} \mathrm{NaCl}$ and supplemented with $10 \mathrm{mM}$ glycerol. Lane 1, W303-1A (wt); Lane 2, BHY29-2C transformed with pRS316 (vector without insert). Lane 3, BHY29-2C transformed with pBH2178 (low-copy plasmid with GUP1). Lane 4, BHY29-2C transformed with pBH2198 (high-copy plasmid with GUP1). B, $\mathrm{BHY} 29-2 \mathrm{C}$ was grown at $30^{\circ} \mathrm{C}$ to $\mathrm{OD}_{600} \sim 3$ and diluted to $\mathrm{OD}_{600}=0.01$. A volume of $1 \mathrm{ml}$ was spread on a YPD plate containing $1 \mathrm{M} \mathrm{NaCl}$. A filter disk containing $20 \mu \mathrm{l}$ of $87 \%$ glycerol was placed in the middle of the plate. Panel 1, BHY29-2C transformed with pRS316 (vector without insert); Panel 2, BHY29-2C transformed with pBH2178 (low-copy GUP1); Panel 3, BHY29-2C transformed with pBH2198 (high-copy GUP1). Plates were incubated at $30^{\circ} \mathrm{C}$ for $48 \mathrm{hr}$.

Fig. 4. Complementation of the gupl mutant strain with GUP1. Cells were grown and spotted as described in the legend to Figure 1. Upper panel: Growth on glycerol as carbon and energy source. Lower panel: YPD containing $1 \mathrm{M} \mathrm{NaCl}$ and supplemented with $10 \mathrm{mM}$ glycerol. Lane 1, W303-

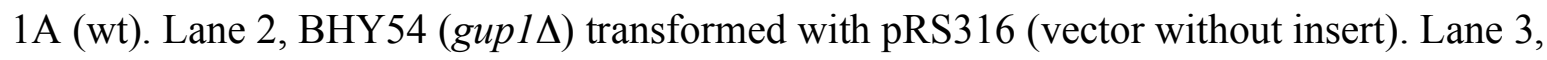
BHY54 transformed with pBH2178 (low-copy plasmid with GUP1). Lane 4, BHY54 transformed with pBH2198 (high-copy plasmid with GUP1). 
Fig. 5. Eadie-Hofstee plots of initial uptake rates of $\left[{ }^{14} \mathrm{C}\right]$ glycerol at $\mathrm{pH} 5.0$ and $30^{\circ} \mathrm{C}$ by $\mathrm{W} 303-1 \mathrm{~A}$

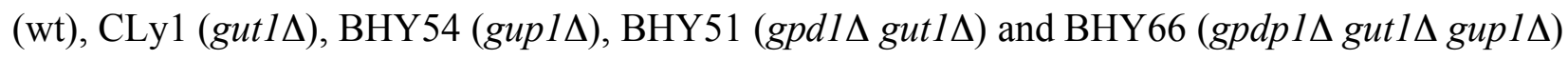
harvested during exponential growth on $2 \%$ ethanol.

Fig. 6. Radio-labelled glycerol accumulation ratios at $\mathrm{pH} 5.0$ and $30^{\circ} \mathrm{C}$ in $\mathrm{W} 303-1 \mathrm{~A}$ (wt), $\mathrm{BHY} 54$

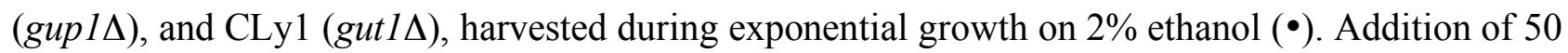
$\mu \mathrm{M} \mathrm{CCCP}$ as indicated by an $\rightarrow(\mathrm{O})$. Incubation in the presence of $50 \mu \mathrm{M} \mathrm{CCCP}(\Delta)$.

Fig. 7. Subcellular localisation of Gup1p as investigated by sucrose gradient centrifugation. Total membranes from a protease-deficient yeast strain (W3124) transformed with a Gup1p-HAexpressing plasmid were isolated and separated on a sucrose gradient. Fractions were collected and analysed for the activity of (open circles) plasma membrane $\mathrm{H}^{+}$-ATPase activity (A), endoplasmic reticulum NADPH-cytochrome oxidase (B), mitochondrial cytochrome oxidase (C) and vacuolar $\alpha$-mannosidase (D). The distribution of Gup1p was determined by Western blotting and quantification of the bands (closed circles). The triangular symbols in panel D represent the total protein concentration in $\mathrm{mg} / \mathrm{ml}$. 


\section{Acknowledgements}

R. Ansell and S. Hohmann (Göteborg University, Sweden) are gratefully acknowledged for communicating the observation that formed the basis for the plate screen for osmotic protection by added glycerol. Thanks are also due to J. Heinisch (Heinrich-Heine-Universität Dusseldorf, Germany), S. Hohmann, A.-K. Påhlman and L. Adler (Göteborg University) and J.R. Winther (Carlsberg Laboratory) for the gift of yeast strains and to M. Snyder (Yale University, USA) for kind provision of the mTn-lacZ/LEU2- mutagenised library. M. Tamás (Katholieke Universiteit, Leuven, Belgium) and P. Philippsen (Basel University, Switzerland) are acknowledged for kind provision of plasmids. This work was carried out according to contract BIO4-CT95-0161 with the European Community, DG12, Framework IV, Biotechnology, Cell Factories. 


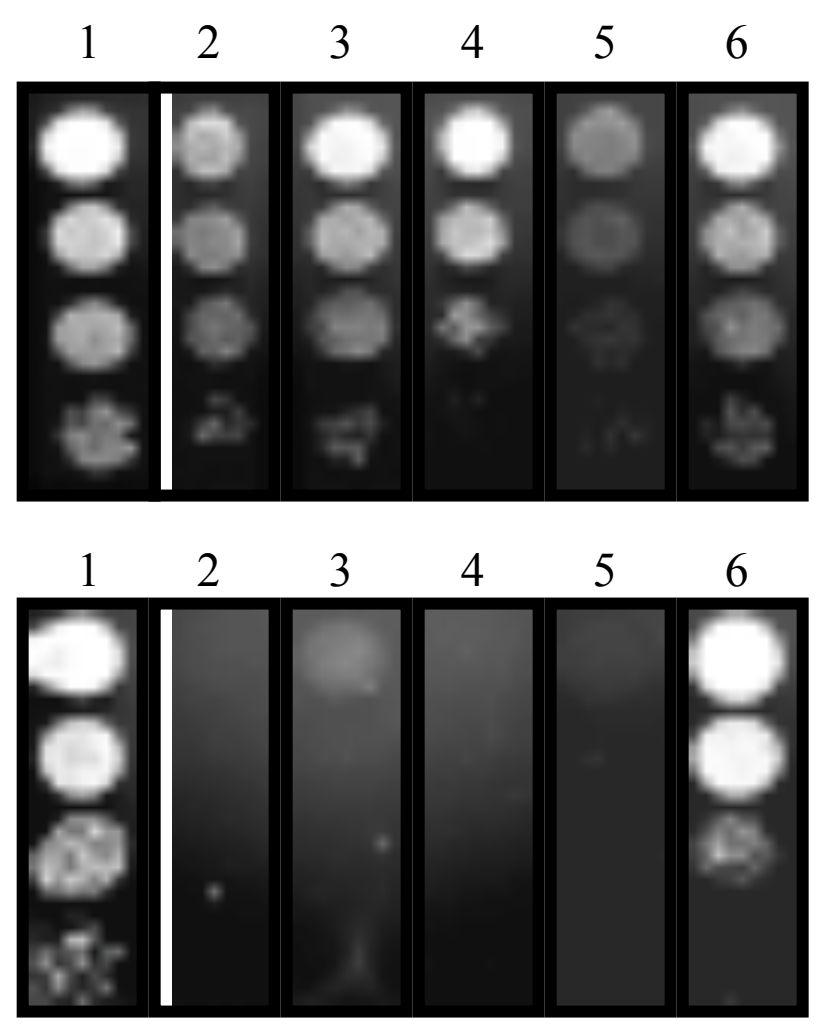




\section{A}

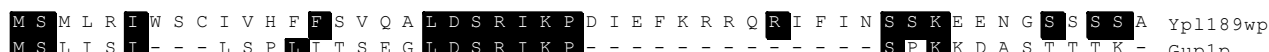

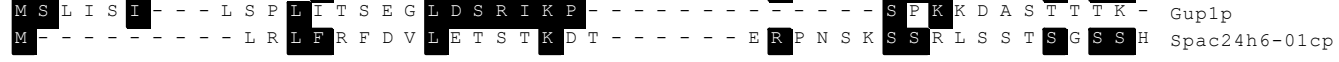

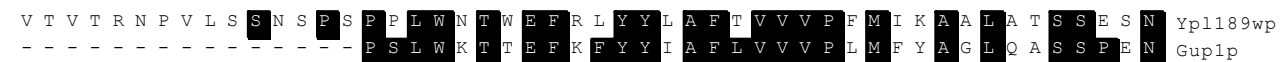

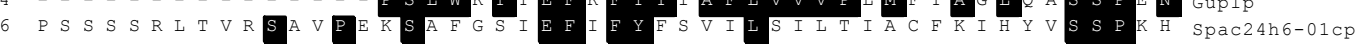

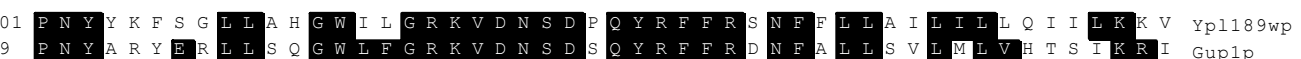

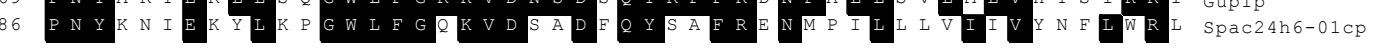

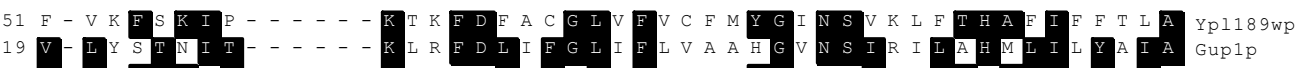

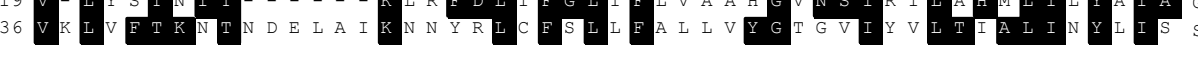

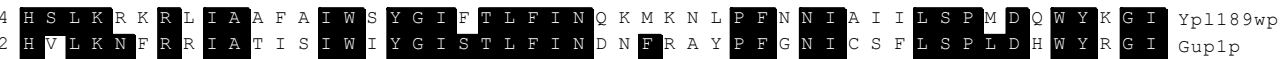

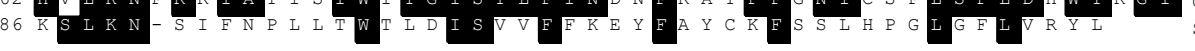

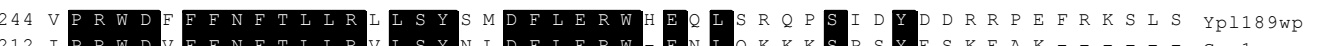

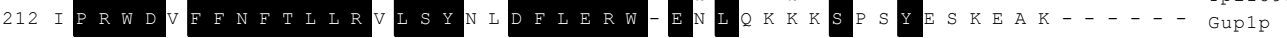

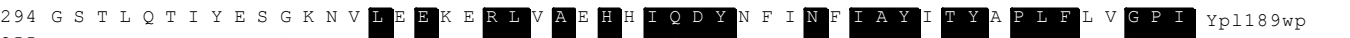
${ }_{213}^{250}$ 344 I T F N D P Y L Y Q S E N K L P S L T T K K K N I G F Y A L K V F S S L L L M M E I I I L H Y I

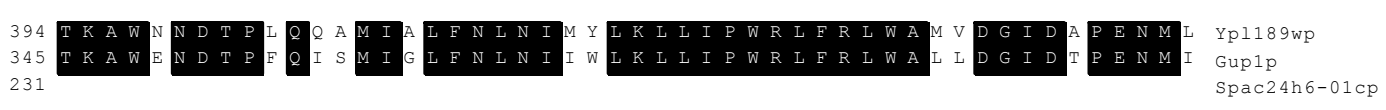

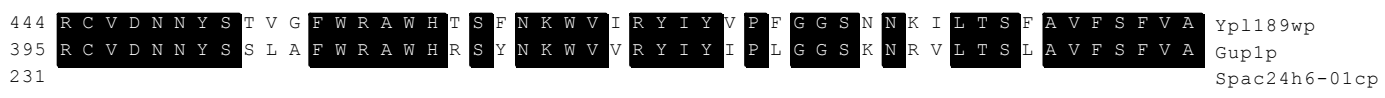

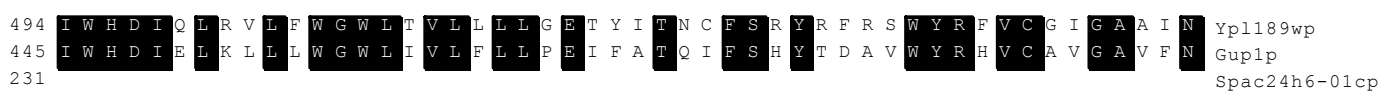

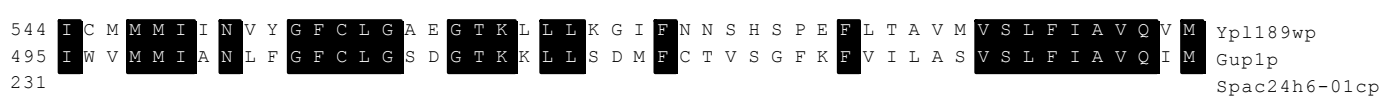
$\begin{array}{lllllllllllllll}594 & \text { F E I R E E E K R H G I N L K C } & & \text { Ypl189wp } \\ 545 & \text { F E I R R E E E K R H G I Y L K C } & \text { Gup1p } \\ 231 & & & & \end{array}$

\section{B}

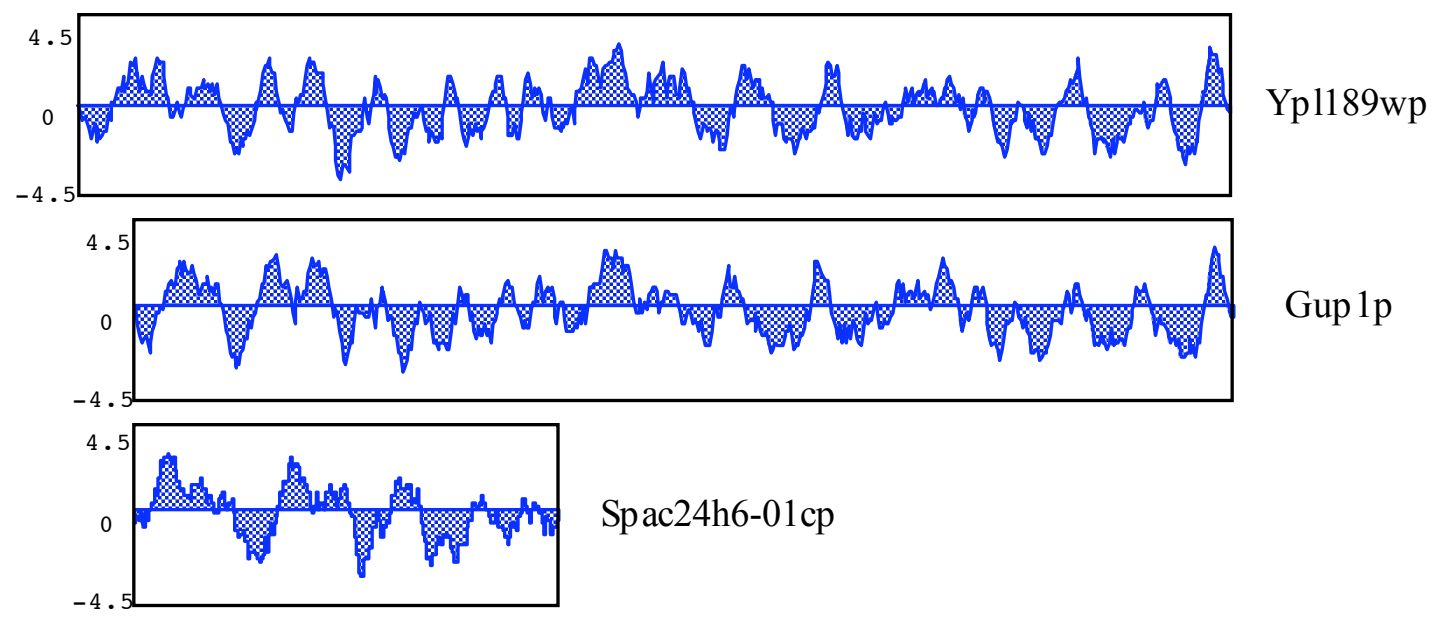




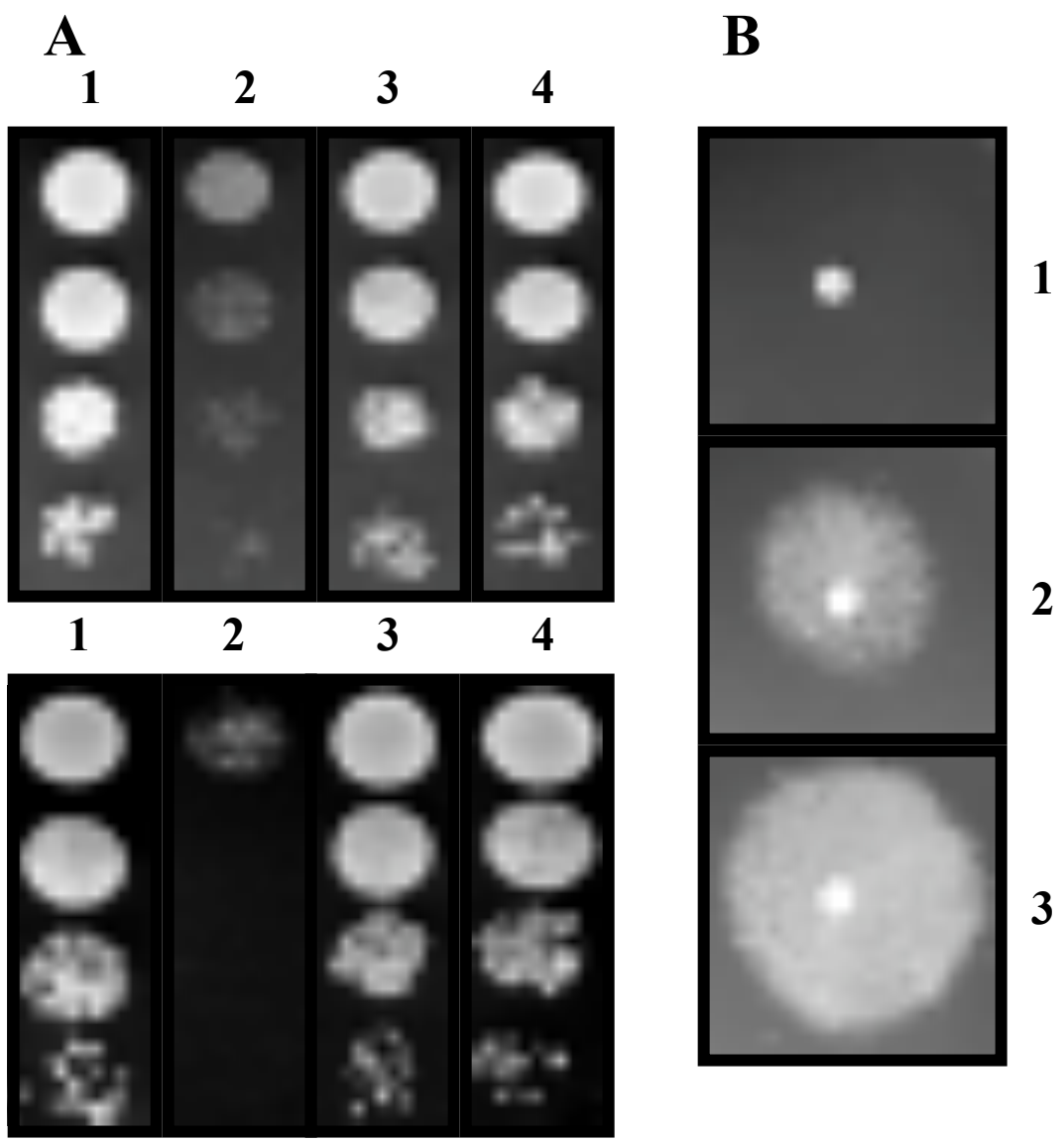




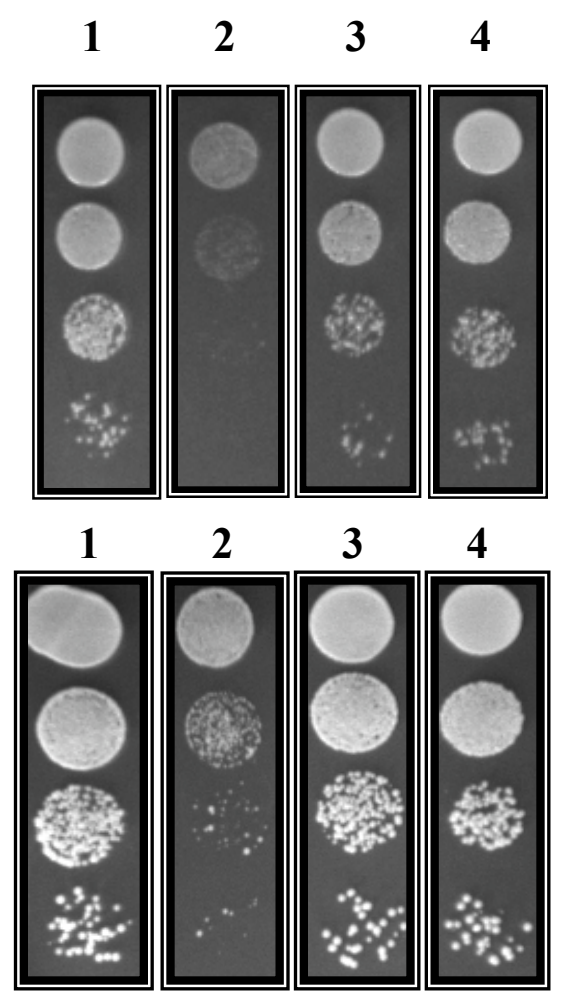



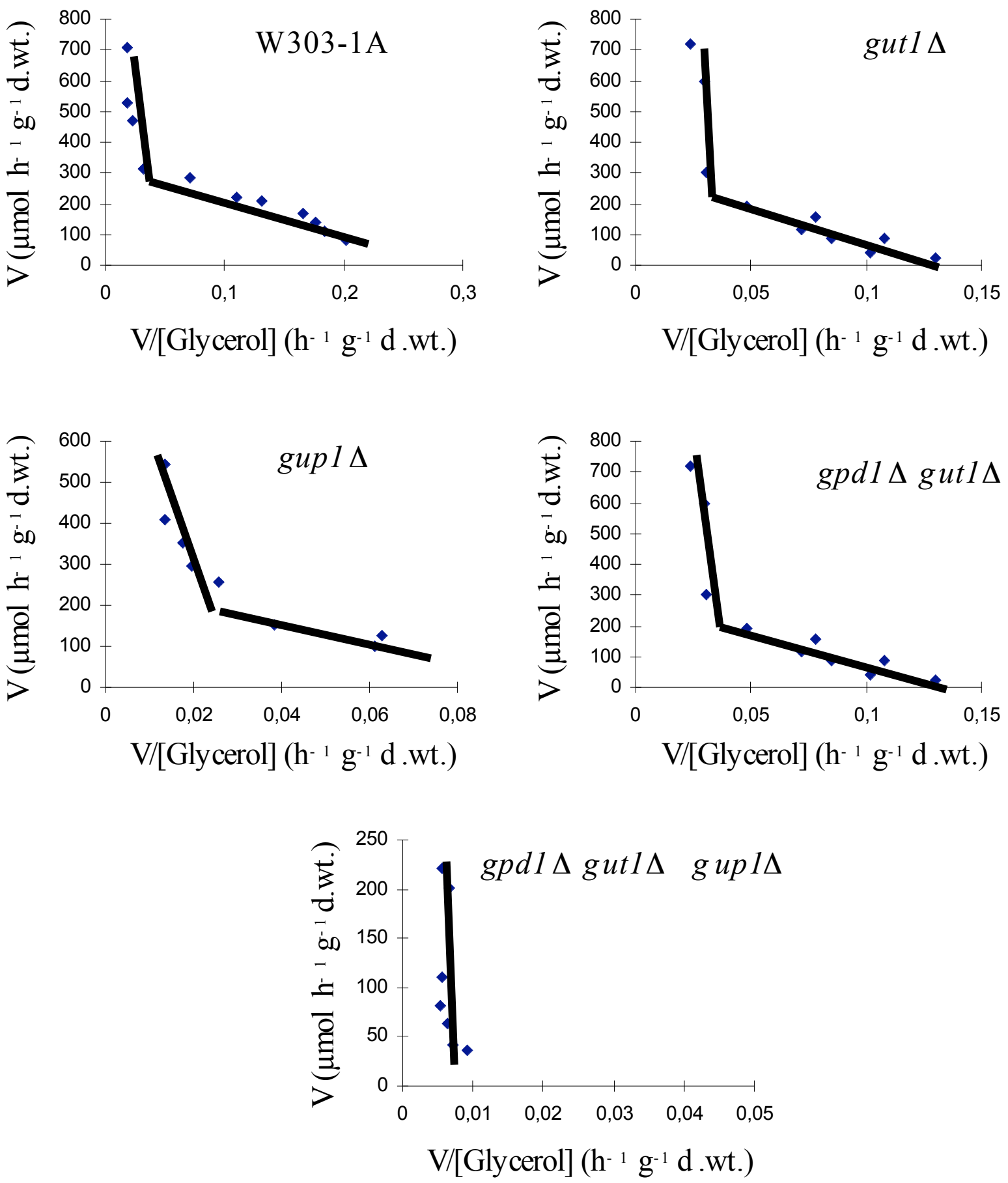

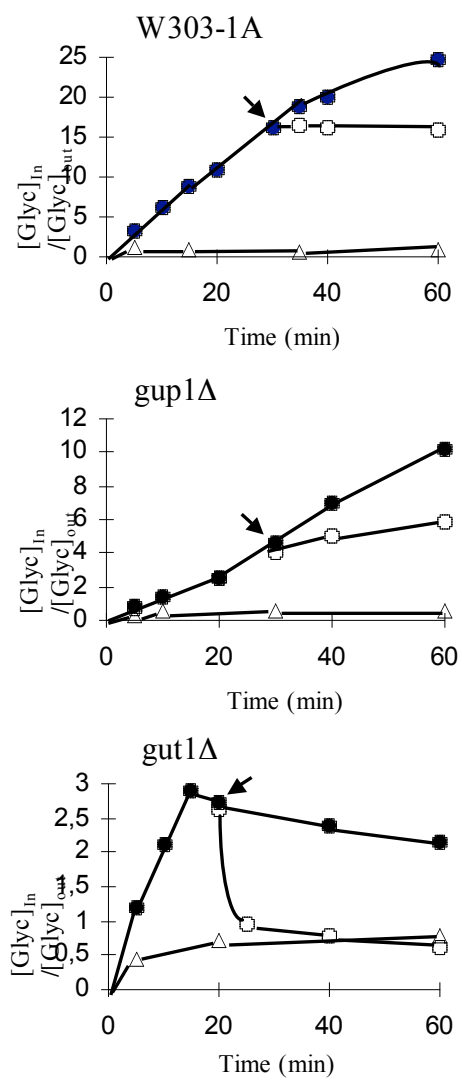
A
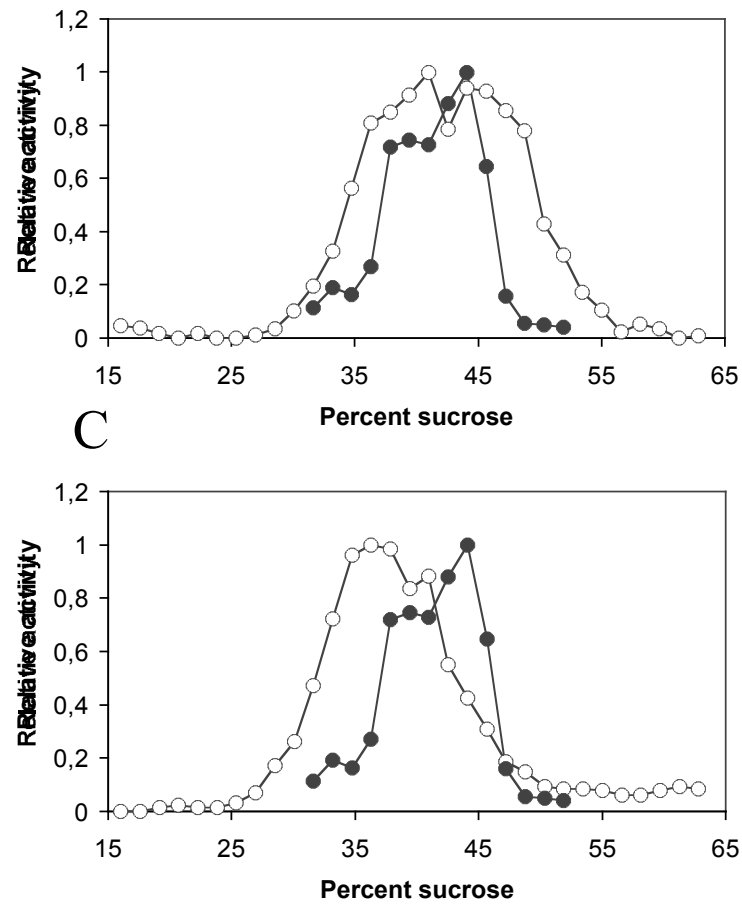

B

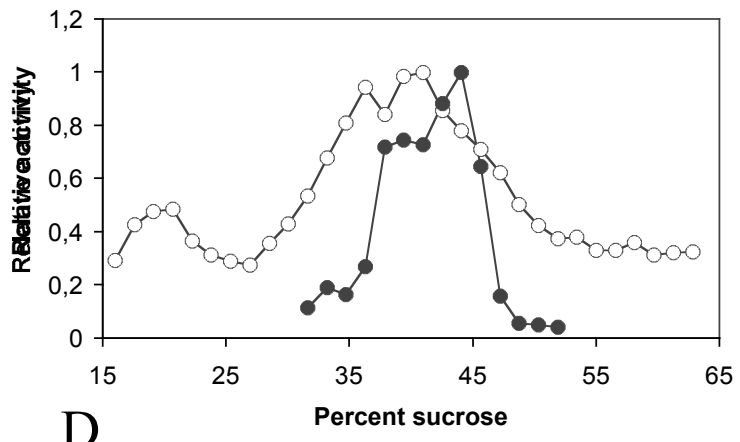

D

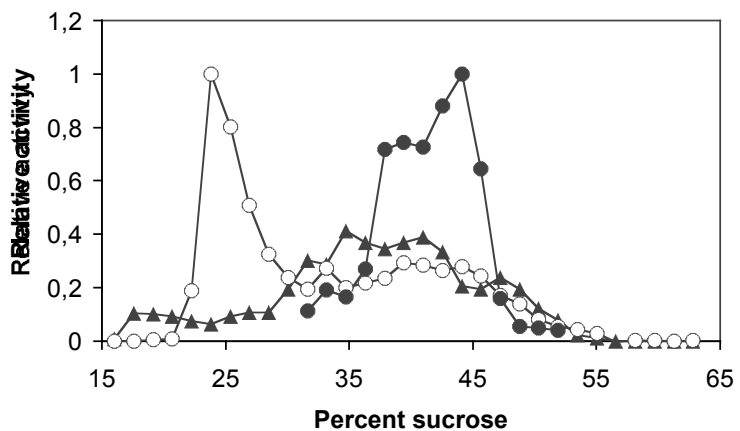

